\title{
GENERALIZED EULER CHARACTERISTICS, GRAPH HYPERSURFACES, AND FEYNMAN PERIODS
}

\author{
PAOLO ALUFFI
}

\begin{abstract}
We give a very informal presentation of background on the Grothendieck group of varieties and on characteristic classes, both viewed as generalizations of the ordinary topological Euler characteristic. We then review some recent work using these tools to study 'graph hypersurfaces' - a topic motivated by the algebro-geometric interpretation of Feynman amplitudes as periods of complements of these hypersurfaces. These notes follow closely, both in content and style, my lectures at the Summer school in Villa de Leyva, July 5-8, 2011.
\end{abstract}

\section{INTRODUCTION}

These notes collect the material covered in my lectures at the Summer school in Villa de Leyva, and they preserve the very informal style of my presentations. In the first part, the main character in the story is the notion of 'generalized Euler characteristic'. Two examples are presented in some detail: the Grothendieck ring of varieties, and the theory of Chern classes for singular varieties (the Chern-Schwartz-MacPherson classes). In the second part graph hypersurfaces are introduced, with a quick explanation of their relevance in the study of Feynman amplitudes. The topics come together in the third part, which gives a review of some recent work studying Grothendieck classes and Chern classes of graph hypersurfaces.

A gentle warning to the reader: nothing in these notes is new, and much is rough beyond the pale. I have tried to provide enough references to the literature so that a reader who so desires can fill in the large gaps left open here. As my audience consisted of both math and physics students, I was not assuming a specific background in algebraic geometry in my lectures, and I am likewise not assuming the same here; this accounts for the need to include fly-by definitions of standard concepts, aimed at providing a bare minimum to be able to make sense of the rest of the notes, and opting for impressionistic blotches of color over detailed photorealism. It goes without saying that any such attempt is doomed to failure, and that at some point in the notes I will have to rely on more background than suggested at the beginning. Nevertheless, I hope that the few rough definitions given in $\$ 2$ may tug the memory of someone who has had previous encounters with algebraic geometry, and facilitate the recall needed for the rest.

The more substantial part of the notes (4) summarizes joint work with Matilde Marcolli (especially AM11a, AM11c, AM11c]). A much more detailed review of this material, including a more extensive description of the physics context which originally motivated it, may be found in Mar10.

Acknowledgments. I thank Alexander Cardona, Hernán Ocampo, Sylvie Paycha, and Andrés Reyes for the invitation to lecture at the summer school on Geometric, Algebraic, and Topological Methods for Quantum Field Theory. The collegial and relaxed atmosphere of the school, the interaction with the students and the other lecturers, and the intensely beautiful surroundings made this a wonderful experience. I also thank Ellie Abernethy, whose notes taken at my lectures simplified greatly the task of putting together this material. 


\section{Generalized Euler characteristics}

2.1. The Euler characteristic. Throughout these notes, we will work with complex quasiprojective algebraic varieties. Reminder on terminology:

- An affine algebraic set is the set of zeros of a collection of polynomials in $\mathbb{C}\left[x_{1}, \ldots, x_{n}\right]$, viewed as a subset of affine space $\mathbb{A}_{\mathbb{C}}^{n}\left(=\mathbb{C}^{n}\right)$.

-A projective algebraic set is likewise the set of zeros of a collection of homogeneous polynomials in $\mathbb{C}\left[z_{0}, \ldots, z_{n}\right]$, viewed as a subset of projective space $\mathbb{P}^{n}=\mathbb{P}_{\mathbb{C}}^{n}$.

Algebraic subsets of a given algebraic set are the closed sets of a topology, called the Zariski topology. An algebraic set is a variety if it is irreducible, that is, it cannot be written as a union of two proper closed subsets (i.e., two proper algebraic subsets). Adhering to an unfortunate but common abuse of language, we may use the term 'variety' for sets which are not necessarily irreducible.

-A quasi-projective variety is a Zariski-open subset of a projective variety, i.e., a set which may be written as $Y \backslash Z$ where both $Y$ and $Z$ are projective.

Quasi-projective varieties admit open coverings by affine varieties. Morphisms of varieties are regular maps, i.e., function which restrict to polynomial functions on members of an affine cover. A birational isomorphism $X \rightarrow Y$ is the datum of an isomorphism between dense open subsets of $X$ and $Y ; X$ and $Y$ are then said to be 'birationally isomorphic' or 'birationally equivalent'. A hypersurface of a variety is an algebraic subset which can de defined locally by a single equation. A variety is smooth, or nonsingular, if it is a manifold. This can be checked easily in terms of the equations defining the variety. For example, a hypersurface $X$ of $\mathbb{A}^{n}$ given by $f\left(x_{1}, \ldots, x_{n}\right)=0$ is nonsingular at a point $p$ if the gradient $\nabla_{p} f$ is not zero; the linear form defined by $\nabla_{p} f$ is the tangent space to $X$ at $p$. If a variety $X$ is nonsingular at all of its points, the collection of the tangent spaces forms the tangent bundle $T X$ to the variety. If $Z$ is a nonsingular subvariety of a nonsingular variety $X$, then $Z$ carries a normal bundle $N_{Z} X$, that is, the quotient $\left.T X\right|_{Z} / T Z$. The blow-up of a variety $X$ along a proper closed subvariety $Z$ is a morphism which restricts to an isomorphism over the complement $X \backslash Z$ (so that blow-ups are birational), and replaces $Z$ by an exceptional divisor, which is a copy of the projectivized normal bundle to $Z$ in $X$ if both $X$ and $Z$ are nonsingular. According to H. Hironaka's famous theorem on resolution of singularities ([Hir64]), every variety is birationally isomorphic to a nonsingular variety, and the resolution may be achieved by a sequence of blow-ups at smooth centers.

Definition 2.1. The (topological) Euler characteristic of $X$ is the alternating sum $\chi(X):=$ $\sum_{i}(-1)^{i} \operatorname{dim} H_{c}^{i}(X, \mathbb{Q})$.

Here $H_{c}$ denotes cohomology with compact support. As it happens, every complex algebraic variety may be compactified within its homotopy type by giving it a topological boundary with odd-dimensional strata. This implies in particular that the compactly supported Euler characteristic equals the ordinary Euler characteristic. (See e.g., [Ful93], p. 95 and 141-2.)

As every projective variety $X$ may be triangulated ([Hir75]), we can also define the Euler characteristic as

$$
\chi(X)=s_{0}-s_{1}+s_{2}-\cdots
$$

where $s_{i}$ is the number of real-dimension $i$ simplices in a triangulation. If $X$ is quasiprojective, say realized as $X=Y \backslash Z$ with $Y$ and $Z$ projective, then $\chi(X)=\chi(Y)-\chi(Z)$ independently of the realization.

The following properties are easy consequences of the definition:

(i) If $X$ and $X^{\prime}$ are isomorphic, then $\chi(X)=\chi\left(X^{\prime}\right)$; 
(ii) If $Z \subseteq X$ is a closed subvariety, then $\chi(X)=\chi(Z)+\chi(X \backslash Z)$;

(iii) $\chi(X \times Y)=\chi(X) \cdot \chi(Y)$.

For us, a 'generalized Euler characteristic' is an invariant satisfying the same properties. In this section we will look at two examples: invariants obtained from homomorphisms from the Grothendieck group/ring of varieties, and the Chern-Schwartz-MacPherson class.

Note that a formal consequence of (ii) is an inclusion-exclusion principle for the Euler characteristic: if $Y$ and $Z$ are subvarieties of a variety $X$, then

$$
\chi(Y \cup Z)=\chi(Y)+\chi(Z)-\chi(Y \cap Z) .
$$

As a consequence of (iii) (and of inclusion-exclusion), the reader can verify that if $X \rightarrow Y$ is locally trivial, with fiber $F$, then $\chi(X)=\chi(Y) \cdot \chi(F)$.

2.2. $K_{0}$ (Var). Quasi-projective varieties are objects of a category Var, with regular maps as morphisms. We are still taking the base field to be $\mathbb{C}$, but it is useful to consider other possibilities, such as $\mathbb{Q}, \mathbb{F}_{q}$ (the finite field with a prime power $q$ of elements). In fact, it is very useful to consider the case of $\mathbb{Z}$ as ground ring, and this will occasionally play a role in what follows.

We are going to define a group $K_{0}$ (Var), called the 'Grothendieck group of varieties'. As a motivation for the name, recall that the Grothendieck group of the category of (say, finitely generated) modules over a ring $R$ is defined as the quotient of the free abelian group on isomorphism classes of $R$-modules by a relation $[M]=\left[M^{\prime}\right]+\left[M^{\prime \prime}\right]$ for every exact sequence $0 \rightarrow M^{\prime} \rightarrow M \rightarrow M^{\prime \prime} \rightarrow 0$. More generally, the same concept may be defined for every small abelian category. It would be very convenient if we could define a similar object for a category such as Var, but we cannot use the same strategy, as Var is not an abelian category. There is a 'difficult' approach to this obstacle: define a suitable enhancement of Var which makes it an abelian category of 'motives', and then consider the Grothendieck group of this category. Defining a good category of motives is in fact an extremely worthwhile goal, but there are very substantial difficulties involved in the construction, and the whole topic is well beyond the scope of these notes. For example, one does not expect the result to be an abelian, rather a triangulated category; relations in the corresponding Grothendieck group arise from triangles. The reader may consult [Lev08] for a general review, and Chapter 2 in [Mar10] for a treatment very close to the context of these notes. The situation is more manageable for 'pure' motives, i.e., motives of smooth projective varieties. Also, motives come in different flavors; 'Chow' motives are based on an equivalence that is especially germane to algebraic geometry.

In any case, in this category there ought to be a distinguished triangle with vertices $Z$, $X$, and $X \backslash Z$ for every variety $X$ and every closed subvariety $Z \subset X$. This suggests an accelerated path to the definition of a 'Grothendieck group of varieties'.

\section{Definition 2.2.}

$$
K_{0}\left(\operatorname{Var}_{k}\right):=\frac{\text { Free abelian group on the set of isom. classes of quasi-proj. } k \text {-varieties }}{\langle[X]=[Z]+[X \backslash Z] \text { for every closed embedding } Z \subseteq X\rangle}
$$

is the Grothendieck group of $k$-varieties. This group is a ring with the operation defined on generators by $[X] \cdot[Y]=\left[X \times_{k} Y\right]$.

The Grothendieck class (or naive motive) of a variety $X$ is its class in $K_{0}\left(\operatorname{Var}_{k}\right)$.

I will usually (but not always) take $k=\mathbb{C}$, and omit the subscript $k$ in the notation. The good news now is that this simple-minded object carries at least as much information as the Grothendieck group obtained from the more involved theory of motives: 
Fact: There is a ring homomorphism from $K_{0}$ (Var) to the Grothendieck ring of pure Chow motives, assigning to the Grothendieck class $[X]$ the class of the Chow motive of $X$.

In this sense we view $[X]$ as a 'naive' counterpart to the motive of $X$. The fact was proven by H. Gillet and C. Soulé ([GS96]), by F. Guillén and V. Navarro Aznar ([GNA02]), and recovered by F. Bittner from a very useful alternative description of $K_{0}$ (Var) ([Bit04]) which will be recalled below.

Theorem 2.3. Let $R$ be a ring and $e$ an $R$-valued invariant of quasi-projective varieties such that

- If $p$ is a point, then $e(p)=1$;

- If $X$ and $X^{\prime}$ are isomorphic, then $e(X)=e\left(X^{\prime}\right)$;

- If $Z \subseteq X$ is a closed subvariety, then $e(X)=e(Z)+e(X \backslash Z)$;

- $e(X \times Y)=e(X) e(Y)$.

Then e is induced by a unique ring homomorphism $K_{0}(\operatorname{Var}) \rightarrow R$.

Theorem 2.3 is essentially tautological: the construction of the Grothendieck ring of varieties parallels the construction of many other objects satisfying a universal requirement, and Theorem 2.3 does nothing but spell out this universal requirement in the case of $K_{0}$ (Var).

It should now be clear that, by Theorem 2.3 , the Euler characteristic $\chi$ defined in $\$ 2.1$ is induced by a unique ring homomorphism $K_{0}$ (Var) $\rightarrow \mathbb{Z}$, and that the ring-valued invariants satisfying our loose requirement for being 'generalized Euler characteristics' are precisely the invariants induced by ring homomorphisms from $K_{0}$ (Var). The assignment sending a variety $X$ to its Grothendieck class $[X] \in K_{0}$ (Var) is a 'universal Euler characteristic' in this sense.

There are other interesting ring homomorphisms naturally defined on $K_{0}$ (Var): as mentioned above, there is a homomorphism from $K_{0}$ (Var) to the Grothendieck ring of pure Chow motives: so the class of a variety in this ring is a 'generalized Euler characteristic'. As another example, the 'Hodge-Deligne polynomial', recording ranks of mixed Hodge structures, takes values in a polynomial ring $\mathbb{Z}[u, v]$. Also: for varieties defined over $\mathbb{Z}$ and every prime power $q$ we can define homomorphisms $K\left(\operatorname{Var}_{\mathbb{Z}}\right) \rightarrow \mathbb{Z}$ by sending $[X]$ to the number of points of $X$ over the finite field $\mathbb{F}_{q}$ (i.e., the number of solutions of the corresponding equations in the finite field $\mathbb{F}_{q}$ ). This latter type of invariants will be of interest to us in 33.2 , see also Example 2.5 below.

The reader is addressed to [Bit04] for an excellent treatment of the universal Euler characteristic. In this paper, F. Bittner gives a very useful alternative presentation for $K_{0}$ (Var). I have recalled that if $X$ is nonsingular and $Z \subset X$ is a nonsingular subvariety, then there exists a 'blow-up' morphism $\widetilde{X} \rightarrow X$ which is an isomorphism over $X \backslash Z$; the inverse image $E$ of $Z$ is the 'exceptional divisor' of this blow-up. It follows that

$$
[\tilde{X}]-[E]=[X]-[Z]
$$

in $K_{0}$ (Var). Bittner proves that $K_{0}$ (Var) is generated by the classes of smooth projective varieties, subject to the relations (1).

Therefore, in order to define a 'generalized Euler characteristic' with values in a ring, it suffices to define it for smooth projective varieties, and prove that the definition is compatible with the blow-up relations (1) (and with multiplicativity). This is often much easier to handle than the scissor-type relations $[X]=[Z]+[X \backslash Z]$ used in Definition 2.2.

Definition 2.4. The Lefschetz (naive) motive is the Grothendieck class $\mathbb{L}:=\left[\mathbb{A}^{1}\right]$ of the affine line. Elements of the subring $\mathbb{Z}[\mathbb{L}] \subseteq K_{0}$ (Var) are called mixed-Tate (naive) motives. 
The (not very standard) terminology introduced here reflects the fact that classes in $\mathbb{Z}[\mathbb{L}]$ correspond to mixed-Tate motives in the category of motives. (For the sake of simplicity of exposition I am neglecting a localization that is in principle needed here.) The reader is addressed to [Mar10], $\S \S 2.5-6$, for more information on mixed-Tate motives. In practice, we view a variety whose Grothendieck class is a naive mixed-Tate motive as 'decomposable' as a disjoint union of affine cells. The reader should verify that, for example,

$$
\left[\mathbb{P}^{n-1}\right]=1+\mathbb{L}+\cdots+\mathbb{L}^{n}=\frac{\mathbb{L}^{n}-1}{\mathbb{L}-1}
$$

is in $\mathbb{Z}[\mathbb{L}]$, and that that if $X$ is nonsingular, $Z \subseteq X$ is a closed nonsingular variety, and $\widetilde{X}$ is the blow-up of $X$ along $Z$, then $[\widetilde{X}]$ is in $\mathbb{Z}[\mathbb{L}]$ if $[X]$ and $[Z]$ are in $\mathbb{Z}[\mathbb{L}]$. As we will see in $\$ 3$, the question of whether the class of a variety is in $\mathbb{Z}[\mathbb{L}]$ can be very interesting and challenging; 'graph hypersurfaces' provide lots of examples, but as we will see not all graph hypersurfaces will be mixed-Tate.

Example 2.5. To get right away a feeling about how 'special' it is for a variety to be mixedTate, note that if $[X] \in \mathbb{Z}[\mathbb{L}] \subseteq K_{0}\left(\operatorname{Var}_{\mathbb{Z}}\right)$, say

$$
[X]=a_{0}+a_{1} \mathbb{L}+\cdots+a_{r} \mathbb{L}^{r},
$$

then the number of points of $X$ over the finite field $\mathbb{F}_{q}$ must be

$$
N_{q}(X)=a_{0}+a_{1} q+\cdots+a_{r} q^{r},
$$

a polynomial in $q$. We will say that $X$ is polynomially countable; we will come back to this in $\$ 3.2$ in the context of graph hypersurfaces.

It is amusing to observe that as the Euler characteristic of the affine line is 1, if the class of $X$ is given by (2), then necessarily $\chi(X)=\sum_{i} a_{i}$ : the value obtained by formally replacing $q$ by 1 in (3). The Euler characteristic of a mixed-Tate motive is $[X]$ is the "number of points of $X$ over $\mathbb{F}_{1}$ '.

2.3. $c_{\text {SM }}$ classes. Notwithstanding the universality of the Grothendieck ring of varieties, homomorphisms of $K_{0}(\mathrm{Var})$ do not exhaust all 'generalized Euler characteristics'. Indeed, the invariants mentioned in Theorem 2.3 have a fixed target $R$, while there is a notion satisfying an inclusion-exclusion principle and (in a suitable sense) multiplicativity, but with target which itself depends on the variety. This is the Chern-Schwartz-MacPherson class, which I will denote by $c_{\mathrm{SM}}(X)$. It takes values in the homology of $X$, or more properly in the Chow group $A_{*} X$ of $X$. This is not the place to discuss rational equivalence and the Chow group in any generality, particularly as we will only be interested in Chern-SchwartzMacPherson classes of specific subsets of projective space; but the reader will bear with us for a moment as we summarize the more general context underlying these classes. (A full treatment of intersection theory is given in Ful84a; the hurried reader will benefit from consulting $\left[\mathrm{Ful} 84 \mathrm{~b}\right.$.) In the case of projective space, $A_{*} \mathbb{P}^{n}$ agrees with homology, and equals the free abelian group on generators $\left[\mathbb{P}^{0}\right], \ldots,\left[\mathbb{P}^{n}\right]$. It is unfortunate that the notation for classes in $A_{*} X$ matches the notation for Grothendieck classes; hopefully the context will clarify the meaning of the notation.

If $X$ is nonsingular, then it carries a tangent bundle $T X$, as already mentioned in 92.1 ; the rank of this bundle is $r=\operatorname{dim} X$. Now, every vector bundle $\mathscr{E}$ on a variety $X$ determines a choice of elements of $A_{*} X$, the Chern classes of $\mathscr{E}$ ([Ful84a], Chapter 3 ). It is common to collect all Chern classes in a 'total Chern class'

$$
c(\mathscr{E})=1+c_{1}(\mathscr{E})+c_{2}(\mathscr{E})+\cdots+c_{\mathrm{rk} \mathscr{E}}(\mathscr{E})
$$


For our purposes, we will rely on the intuition that the $i$-th Chern class of $\mathscr{E}$, denoted $c_{i}(\mathscr{E}) \cap[X]$, is a class in codimension $i$ recording information on the subset of $X$ where rk $\mathscr{E}-i+1$ general sections of $\mathscr{E}$ are linearly dependent. Warning: Making this statement precise is no simple matter; for example, $\mathscr{E}$ may have no nontrivial sections whatsoever; here we are simply capturing the gist of Examples 14.4.1 and 14.4.2 in [Ful84a]. According to this rough interpretation, the 'top' Chern class $c_{\operatorname{dim} X}(T X) \cap[X]$ of the tangent bundle of $X$ is a class in codimension $\operatorname{dim} X$ (i.e., in dimension 0 ), recording the locus where a general section of $T X$ (i.e., a general vector field) is linearly dependent (i.e., it vanishes). Now, the Poincaré-Hopf theorem tells us that the (suitably interpreted) number of zeros of a vector field on a compact nonsingular variety equals the Euler characteristic of the variety. Therefore:

Lemma 2.6 (Poincaré-Hopf).

$$
\int c(T X) \cap[X]=\chi(X)
$$

Here, the integral sign $\int$ denotes the degree of the dimension 0 part of the class that follows. Thus, Lemma 2.6 is simply a statement of the Poincaré-Hopf theorem, once we interpret the dimension 0 part of the Chern class of $T X$ as mentioned above. (And again, it should be clear that we are sweeping substantial subtleties under the rug. For example, what if $\chi(X)<0$ ?) A similar statement may be given relating all Chern classes of $T X$ and loci at which there arise obstructions to the construction of frames of vector fields.

A natural question is whether the Poincaré-Hopf theorem (and the companion statements for all Chern classes) can be recovered if $X$ is singular: in this case, $X$ does not carry a tangent bundle (the rank of the tangent space will jump at singular points), so the left-hand side of (4) loses its meaning.

In the 1960s, Marie-Hélène Schwartz answered this question by introducing and studying special vector fields at the singularities of $X$ ([Sch65b, Sch65a]). In independent work, Grothendieck and Deligne proposed a conjectural framework of which Theorem 2.6 is a tiny aspect, later confirmed by R. MacPherson ([Mac74]). Later still, the classes defined by Schwartz and MacPherson were found to agree ([BS81, [AB08]); they are commonly called Chern-Schwartz-MacPherson classes, $c_{\mathrm{SM}}$ classes for short.

In MacPherson's approach, one defines a class $c_{\mathrm{SM}}(\varphi)$ in $A_{*} X$ (MacPherson worked in homology, but the theory may be defined in the Chow group; cf. [Ful84a], Example 19.1.7) for every constructible function on a variety $X$. A constructible function is a linear combination of indicator functions of subvarieties: $\varphi=\sum m_{i} \mathbb{1}_{Z_{i}}$. Constructible functions on $X$ form an abelian group $\mathscr{C}(X)$, and in fact $\mathscr{C}$ is a covariant functor: if $f: X \rightarrow Y$ is a regular map, we can define a homomorphism $\mathscr{C}(X) \rightarrow \mathscr{C}(Y)$ by setting

$$
f_{*}\left(\mathbb{1}_{Z}\right)(p)=\chi\left(f^{-1}(p) \cap Z\right)
$$

for any $p \in Y$ and any subvariety $Z$, and extending this prescription by linearity. MacPherson's definition has the following properties:

- For any $X, c_{\mathrm{SM}}: \mathscr{C}(X) \rightarrow A_{*} X$ is a homomorphism;

- If $X$ is nonsingular and compact, then $c_{\mathrm{SM}}\left(\mathbb{1}_{X}\right)=c(T X) \cap[X]$;

- If $f: X \rightarrow Y$ is a proper morphism and $\varphi \in \mathscr{C}(X)$, then $c_{\mathrm{SM}}\left(f_{*} \varphi\right)=f_{*} c_{\mathrm{SM}}(\varphi)$.

In the third property, $f$ is required to be proper to have a good notion of push-forward of classes in the Chow group. Various extensions of the theory (to more general fields, or allowing for non-proper morphisms) have been considered ([Ken90], [Alu06]). Resolution of singularities and the covariance property of $c_{\mathrm{SM}}$ classes reduce (in principle) the computation 
of any $c_{\mathrm{SM}}$ class to computations for nonsingular varieties, where the $c_{\mathrm{SM}}$ class agrees with the class of the tangent bundle. This also implies that the $c_{\mathrm{SM}}$ natural transformation is uniquely determined by the properties listed above.

The class $c_{\mathrm{SM}}\left(\mathbb{1}_{X}\right)$ is usually denoted $c_{\mathrm{SM}}(X)$, and called the Chern-Schwartz-MacPherson class of $X$. Abusing language, we denote by $c_{\mathrm{SM}}(Y)$ the class $c_{\mathrm{SM}}\left(\mathbb{1}_{Y}\right)$ in $A_{*} X$ for any subvariety (closed or otherwise) of $X$.

What the above properties say is that $c_{\mathrm{SM}}$ is a natural transformation, specializing to ordinary Chern classes in the nonsingular case. To get a feeling for the type of information packed into this statement, consider the extremely special case of the constant function $\kappa: X \rightarrow\{\mathrm{pt}\}$ (where $X$ is assumed to be compact). The push-forward at the level of homology/Chow group,

$$
\kappa_{*}: A_{*} X \rightarrow A_{*}\{\mathrm{pt}\}=\mathbb{Z}
$$

is nothing but the 'integral' $\int$ mentioned above. According to the above properties,

$$
\int c_{\mathrm{SM}}(X)=\kappa_{*} c_{\mathrm{SM}}\left(\mathbb{1}_{X}\right)=c_{\mathrm{SM}}\left(\kappa_{*} \mathbb{1}_{X}\right)=\chi(X) c_{\mathrm{SM}}\left(\mathbb{1}_{\mathrm{pt}}\right)=\chi(X)
$$

as the $c_{\mathrm{SM}}$ class of a point is 1 (times the class of the point). Compare (5) with (4): we have recovered for arbitrary (compact) varieties a 'Poincaré-Hopf theorem'.

Equation (5) already shows that $c_{\mathrm{SM}}(X)$ is a direct generalization of the Euler characteristic. Further, if $Z \subseteq X$, then

$c_{\mathrm{SM}}(X)=c_{\mathrm{SM}}\left(\mathbb{1}_{X}\right)=c_{\mathrm{SM}}\left(\mathbb{1}_{Z}+\mathbb{1}_{X \backslash Z}\right)=c_{\mathrm{SM}}\left(\mathbb{1}_{Z}\right)+c_{\mathrm{SM}}\left(\mathbb{1}_{X \backslash Z}\right)=c_{\mathrm{SM}}(Z)+c_{\mathrm{SM}}(X \backslash Z)$

(adopting the notational convention mentioned above). Thus, $c_{\mathrm{SM}}$ classes satisfy requirement (ii) for 'generalized Euler characteristics' in 2.1 . They also satisfy a form of multiplicativity (requirement (iii)), which we won't describe here, and for which the reader may consult [Kwi92 or [Alu06]. There is a subtlety involved in the first requirement: if $X$ and $X^{\prime}$ are isomorphic, then of course $c_{\mathrm{SM}}(X)$ and $c_{\mathrm{SM}}\left(X^{\prime}\right)$ agree up to the identification $A_{*} X \cong A_{*} X^{\prime}$. However, if $X$ and $X^{\prime}$ are viewed as subvarieties of (for example) projective space, then $c_{\mathrm{SM}}\left(\mathbb{1}_{X}\right)$ and $c_{\mathrm{SM}}\left(\mathbb{1}_{X^{\prime}}\right)$ will depend on the embeddings, and hence may differ. (Thus, the notation $c_{\mathrm{SM}}(X)$ is ambiguous. This is harmless, as long as the embedding of $X$ is clearly specified.)

Example 2.7. - Viewing $\mathbb{P}^{1}$ as a subvariety of itself, $c_{\mathrm{SM}}\left(\mathbb{P}^{1}\right)=\left[\mathbb{P}^{1}\right]+2\left[\mathbb{P}^{0}\right]$ : indeed, since $\mathbb{P}^{1}$ is nonsingular we have

$$
c_{\mathrm{SM}}\left(\mathbb{P}^{1}\right)=c\left(T \mathbb{P}^{1}\right) \cap\left[\mathbb{P}^{1}\right]=\left(1+c_{1}\left(T \mathbb{P}^{1}\right)\right) \cap\left[\mathbb{P}^{1}\right]=\left[\mathbb{P}^{1}\right]+\chi\left(\mathbb{P}^{1}\right)\left[\mathbb{P}^{0}\right]=\left[\mathbb{P}^{1}\right]+2\left[\mathbb{P}^{0}\right]
$$

-More generally,

$$
c_{\mathrm{SM}}\left(\mathbb{P}^{n}\right)=\left[\mathbb{P}^{n}\right]+\left(\begin{array}{c}
n+1 \\
1
\end{array}\right)\left[\mathbb{P}^{n-1}\right]+\left(\begin{array}{c}
n+1 \\
2
\end{array}\right)\left[\mathbb{P}^{n-2}\right]+\cdots+\left(\begin{array}{c}
n+1 \\
n
\end{array}\right)\left[\mathbb{P}^{0}\right]
$$

This again follows from the normalization property for nonsingular varieties, a standard exact sequence for $T \mathbb{P}^{n}$ ([Ful84a, B.5.8), and the Whitney formula for Chern classes. See Example 3.2.11 in Ful84a]. Note that $\int c_{\mathrm{SM}}\left(\mathbb{P}^{n}\right)=\left(\begin{array}{c}n+1 \\ n\end{array}\right)=n+1=\chi\left(\mathbb{P}^{n}\right)$, as it should. A shorthand for (6) is

$$
c_{\mathrm{SM}}\left(\mathbb{P}^{n}\right)=(1+H)^{n+1} \cap\left[\mathbb{P}^{n}\right]
$$

where $H$ is the 'hyperplane class', i.e., $H^{i}$ stands for $\left[\mathbb{P}^{n-i}\right]$. (And in particular $H^{n+1}=0$.)

- On the other hand, a nonsingular conic $C$ in $\mathbb{P}^{2}$ is easily seen to be abstractly isomorphic to $\mathbb{P}^{1}$, but

$$
c_{\mathrm{SM}}(C)=c_{\mathrm{SM}}\left(\mathbb{1}_{C}\right)=c(T C) \cap[C]=\left(1+c_{1}(T C)\right) \cap[C]=2\left[\mathbb{P}^{1}\right]+2\left[\mathbb{P}^{0}\right] .
$$


This agrees with $c_{\mathrm{SM}}\left(\mathbb{P}^{1}\right)$ through the isomorphism, but the result of the push-forward to $\mathbb{P}^{2}$ differs from the class $c_{\mathrm{SM}}\left(\mathbb{P}^{1}\right)$ recorded above.

-A union $X$ of two distinct lines $L_{1}, L_{2}$ in $\mathbb{P}^{2}$ is a singular conic. By inclusion-exclusion, $c_{\mathrm{SM}}(X)=c_{\mathrm{SM}}\left(L_{1} \cup L_{2}\right)$ is given by

$$
c_{\mathrm{SM}}\left(L_{1}\right)+c_{\mathrm{SM}}\left(L_{2}\right)-c_{\mathrm{SM}}\left(L_{1} \cap L_{2}\right)=2 c_{\mathrm{SM}}\left(\mathbb{P}^{1}\right)-c_{\mathrm{SM}}\left(\mathbb{P}^{0}\right)=2\left[\mathbb{P}^{1}\right]+3\left[\mathbb{P}^{0}\right] .
$$

Notice that the $c_{\mathrm{SM}}$ class 'sees' the difference between singular and nonsingular curves of the same degree. In general, $c_{\mathrm{SM}}$ classes may be used to express the Milnor number of a singularity, and more general Milnor classes, see e.g., [PP01. Indeed, one of the main motivations in the study of $c_{\mathrm{SM}}$ classes is as a handle on interesting singularity invariants.

-Exercise for the reader: For subsets of projective space obtained as unions/ intersections/ differences of linear subspaces, the information carried by the $c_{\mathrm{SM}}$ class precisely matches the information carried by the Grothendieck class. The precise statement may be found in AM09, Proposition 2.2.

- In particular, $c_{\mathrm{SM}}$ classes for hyperplane arrangements (and their complement) carry the same information as the corresponding Grothendieck classes. It is not hard to see that this information is the characteristic polynomial of the arrangement (cf. [Alu12]). For instance, for graphical arrangements $c_{\mathrm{SM}}$ classes compute the chromatic polynomial.

For $X \subseteq \mathbb{P}^{n}$, the information carried by $c_{\mathrm{SM}}\left(\mathbb{1}_{X}\right)$ is precisely equivalent to the information of the Euler characteristics of all general linear sections of $X$ ([Alub]).

Work of J.-P. Brasselet, J. Schürmann, S. Yokura treat the $c_{\mathrm{SM}}$ class in terms of a relative Grothendieck group ([BSY10]). For projective sets, there is a group homomorphism from a Grothendieck group associated with $\mathbb{P}^{\infty}$ to the corresponding Chow group, a polynomial ring $\mathbb{Z}[T]$, which yields the $c_{\text {SM }}$ class ([Alub]). The multiplicativity properties of this morphism are interesting, and they will play a role behind the scenes later in these notes (Theorem 4.9).

In any case, the true 'motivic' nature of $c_{\mathrm{SM}}$ classes has likely not yet been understood completely. One of the motivations in carrying out the computations that will be reviewed in the rest of this paper is to collect raw data in interesting situations, with the aim of clarifying possible relations between motives and Chern-Schwartz-MacPherson classes.

\section{Feynman PERIOdS AND GRAPH hyPERSURFACES}

3.1. Feynman periods. In quantum field theory, and more specifically perturbative massless scalar field theories, one is interested in action integrals

$$
S(\phi)=\int \mathscr{L}(\phi) d^{D} x
$$

in dimension $D$, where $\mathscr{L}(\phi)$ is a 'Lagrangian density'. These integrals may be studied by means of a perturbative expansion, which takes the form

$$
S_{\text {eff }}(\phi)=\sum_{G}\langle G\rangle
$$

where the sum ranges over a collection of graphs $G$ determined by the Lagrangian, and the contribution $\langle G\rangle$ of a graph is of the form $\frac{G(\phi)}{\# \operatorname{Aut}(G)}$; we will say more about $G(\phi)$ below. Further manipulations may be used to restrict attention to more special graphs, for example connected, '1-particle irreducible' (1PI) graphs - i.e., graphs without edges ('bridges') whose removal causes $G$ to become disconnected; also see 4.1 . The reader is addressed to any text in quantum field theory (such as [Zee10]) for information on what all of this really means, and to [Mar10] for a more thorough presentation in the same context of this note. 
The contribution $G(\phi)$ due to $G$ is itself an integral, of the form

$$
G(\phi)=\frac{1}{n !} \int_{\sum_{i} p_{i}=0} \hat{\phi}\left(p_{1}\right) \cdots \hat{\phi}\left(p_{n}\right) U\left(G\left(p_{1}, \ldots, p_{n}\right)\right) d p_{1} \cdots d p_{n}
$$

Here $n$ is the number of edges of $G$, and $p_{i}$ denotes a momentum associated with the $i$-th edge. The integration locus is $\sum_{i} p_{i}=0$ to enforce momentum conservation; $\hat{\phi}$ denotes Fourier transform, and the integral is taken over 'momentum space'. This is not the only option: an expression for $G(\phi)$ may be given over 'configuration space', and this turns out to be advantageous for the questions of interest here, as has been understood very recently, see $[\mathrm{CM}]$. In the above integral,

$$
U\left(G\left(p_{1}, \ldots, p_{n}\right)\right)=\int I_{G}\left(k_{1}, \ldots, k_{\ell}, p_{1}, \ldots, p_{n}\right) d^{D} k_{1} \cdots d^{D} k_{\ell},
$$

where $\ell$ is the number of loops in $G$, and the core contribution $I_{G}$ may be written out in an essentially automatic fashion from the combinatorics of $G$, by means of the full set of 'Feynman rules'. We will come back to this in $\$ 4$, where the guiding theme will indeed be the relation between the combinatorics of a graph and the objects of interest to us. But we have not yet described these objects.

Clever use of various reductions (keywords: 'Schwinger parameters', 'Feynman trick') yield an expression for $U(G)$ of the following form:

$$
U(G)=\frac{\Gamma(n-D \ell / 2)}{(4 \pi)^{\ell D / 2}} \int_{\sigma_{n}} \frac{P_{G}(\underline{t}, p)^{-n+D \ell / 2} \omega_{n}}{\Psi_{G}(\underline{t})^{-n+D(\ell+1) / 2}} .
$$

Here the integral is over the (real) symplex $\sigma_{n}=\left\{\sum_{i} t_{i}=1\right\}, \omega_{n}$ is the volume form, and $P_{G}, \Psi_{G}$ are certain polynomials determined by the graph $G$. The reader should take heart in the news that of all the terms introduced so far in this section, this polynomial $\Psi_{G}$ is the only one that will be really relevant to what follows.

There are issues of convergence with all the integrals mentioned here, with which quantum field theory magically manages to deal. For example, the dimension $D$ is used as a parameter in these integrals (occasionally allowing it to become a complex number); here we will pretend that $D$ is large enough that $D \ell / 2-n \geq 0$, so that $P_{G}$ is really at numerator and $\Psi_{G}$ is really at denominator in (8). The Gamma factor $\Gamma(n-D \ell / 2)$ contributes to the divergence of $U(G)$, so one can at best aim at dealing with the residue of $U(G)$, which under the above assumption has the form

$$
\int_{\text {cycle } \sigma} \frac{\text { diff. form }}{\Psi_{G}^{\geq 0}},
$$

an integral of an algebraic differential form over the complement of the hypersurface $\hat{X}_{G}$ defined by $\Psi_{G}=0$. This is the object of interest to us. There are further divergences due to the intersection of $\hat{X}_{G}$ with the domain of integration $\sigma$. Methods have been developed to deal with this, for example sequences of blow-ups which separate the domain from $\hat{X}_{G}$. This is discussed in work of S. Bloch, H. Esnault, D. Kreimer ([BEK06], BK08]), and I will simply gloss over this important issue, for the sake of simplicity, and on the ground that this should not affect the considerations in this paper.

Integrals of algebraic forms over algebraic domains (all defined over $\overline{\mathbb{Q}}$ ) are called periods. The standard reference for this notion is an extensive review article by M. Kontsevich and D. Zagier, [KZ01]. The set of periods forms a ring (often enlarged by including $\frac{1}{2 \pi i}$, for technical reasons), and is countable. It contains all algebraic numbers, but also numbers such as $\pi$; it is surprisingly difficult to prove that a given number is not a period, although 
cardinality ensures that most numbers are not. (For example, $e$ is not believed to be a period, but this is not known.) A period is an expression of the cohomology of a variety, and intuitively varieties with 'simpler' cohomology should have 'simpler' periods. In fact, the periods are determined by the motive of the variety on which they are taken, so they may be seen as an avatar of the motive. For example, periods of mixed-Tate motives are known to be (suitable combinations of) multiple zeta values; this was a long-standing conjecture, and has recently been proved by F. Brown, Bro12]. More precisely: multiple zeta values are defined by

$$
\zeta\left(n_{1}, \ldots, n_{r}\right)=\sum_{0<k_{1}<\cdots<k_{r}} \frac{1}{k_{1}^{n_{1}} \cdots k_{r}^{n_{r}}}
$$

with $n_{i} \geq 1, n_{r} \geq 2$. Brown was able to confirm that all periods of mixed Tate motives over $\mathbb{Z}$ are $\mathbb{Q}\left[\frac{1}{2 \pi i}\right]$-linear combinations of multiple zeta values.

Summarizing: the contributions $U(G)$ may be viewed as certain periods for the (motive corresponding to) the complement $\hat{Y}_{G}$ of the graph hypersurface $\hat{X}_{G} \subseteq \mathbb{A}^{n}$ defined by $\Psi_{G}(\underline{t})=0$. Again, let me point out that there is an issue of divergence (due to the intersection of $\hat{X}_{G}$ with the simplex $\sigma$ over which the integration takes place). In the approach by Bloch et al., this issue is dealt with by means of carefully chosen blow-ups determined by the combinatorics of the graph. While these blow-ups are necessary, their impact on the the motive of the complement should be relatively mild, or so I will assume at any rate. Thus, I will assume that the contributions depend directly on the motive of $\hat{Y}_{G}$.

Now, there are refined numerical techniques that can closely approximate a contribution $U(G)$ for a given graph $G$. Thus, a rather extensive catalogue of examples of these 'Feynman periods' has been obtained with high accuracy. If a number is known with sufficiently high precision, there are techniques that can determine very reliably whether the number is, say, algebraic; or, case in point, whether it is a linear combination of multiple zeta values (with coefficients in $\mathbb{Q}\left[\frac{1}{2 \pi i}\right]$ ). The reference usually quoted for for these computations is [BK97]; the amazing observation stemming from these very extensive computations is that Feynman periods appear to be (combinations of) multiple zeta values. In other words, Feynman periods appear to be periods of mixed Tate motives.

The natural conjecture is then that the complements $\hat{Y}_{G}$ themselves should be mixed Tate motives. Although (as we will see in a moment) this turns out not to be the case, understanding this situation better has motivated a very substantial amount of work, and my goal in $\$ 4$ will be to review a tiny portion of this work. I should point out right away that due to work of F. Brown again ([Bro], Bro09], etc.), and several others, the available physicists' evidence linking Feynman periods to multiple zeta values has been explained, but the general question of whether Feynman periods are always necessarily combinations of multiple zeta values is still open.

3.2. Graph hypersurfaces. The main character of the story has now been identified as the hypersurface given by the vanishing of the 'graph polynomial $\Psi_{G}$ ' mentioned in 3.1 . This polynomial is

$$
\Psi_{G}\left(t_{1}, \ldots, t_{n}\right):=\sum_{T} \prod_{e \notin T} t_{e}
$$

where $T$ ranges over the maximal spanning forests of $G$, and $t_{e}$ is a variable associated with the edge $e$. If $G$ is connected, maximal spanning forests are spanning trees; the reader should note that other references simply set this polynomial to 0 if $G$ is not connected, but we find that it is worth considering $\Psi_{G}$ for arbitrary graphs.

Example 3.1. The spanning trees for the '3-banana graph' 


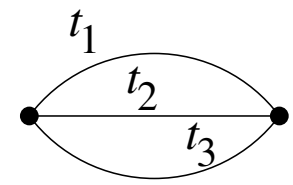

are
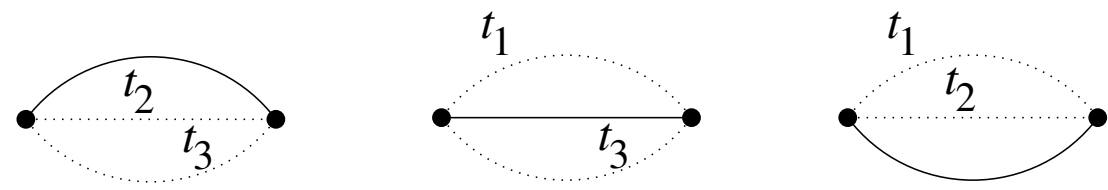

and it follows that

$$
\Psi_{G}(\underline{t})=t_{2} t_{3}+t_{1} t_{3}+t_{1} t_{2}=t_{1} t_{2} t_{3}\left(\frac{1}{t_{1}}+\frac{1}{t_{2}}+\frac{1}{t_{3}}\right)
$$

in this case. Similarly, the graph polynomial for the $n$-banana graph $G$ is

$$
\Psi_{G}(\underline{t})=t_{1} \cdots t_{n}\left(\frac{1}{t_{1}}+\cdots+\frac{1}{t_{n}}\right)
$$

for all $n>0$.

Example 3.2. There are eight spanning trees for the graph

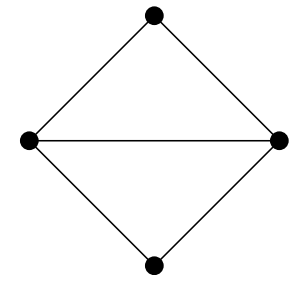

namely

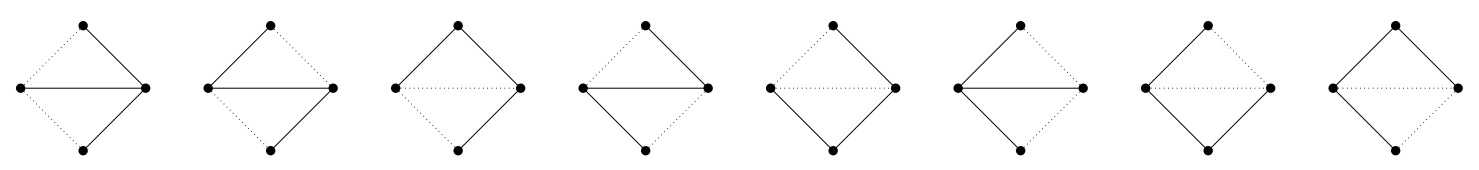

The corresponding graph polynomial is (up to renumbering the edges)

$$
t_{1} t_{2}+t_{1} t_{3}+t_{1} t_{5}+t_{2} t_{4}+t_{2} t_{5}+t_{3} t_{4}+t_{3} t_{5}+t_{4} t_{5}
$$

an irreducible quadratic polynomial.

In the graph theory literature, $\Psi_{G}$ is called the (Kirchoff-Tutte)-Symanzik polynomial. The number of edges in a maximal spanning forest for $G$ is the same for all such forests (exercise!), therefore $\Psi_{G}(\underline{t})$ is homogeneous. Thus, its vanishing defines a hypersurface $X_{G}$ in $\mathbb{P}^{n-1}(n=$ number of edges of $G)$, or equivalently a cone $\hat{X}_{G}$ in $\mathbb{A}^{n}$. We will denote by $Y_{G}$ the complement of $X_{G}$ in $\mathbb{P}^{n-1}$, and by $\hat{Y}_{G}$ the complement of $\hat{X}_{G}$ in $\mathbb{A}^{n}$. Of course each of these objects carries essentially the same information. It is clear from the definition that the degree of $\Psi_{G}$ equals the number of loops $b_{1}(G)$ of $G$.

For very small graphs, $X_{G}$ may be nonsingular: in the example of a 3-banana given above, the corresponding $X_{G}$ is a nonsingular conic in $\mathbb{P}^{2}$. But $X_{G}$ is singular for larger graphs. The reader can verify that the singular locus of $X_{G}$ is defined by (cones over) graph hypersurfaces determined by smaller graphs $G \backslash e$. The study of singularities of $X_{G}$ was initiated by E. Patterson ([Pat10]); some of our own work reviewed in $\$ 4$ is aimed at the computation of global invariants of the singularities of $X_{G}$ analogous to the Milnor 
number. We will also see later on that $X_{G}$ is rational when it is irreducible; and it is known (see for example [SMJŌ77], Proposition 5.2) that $\Psi_{G}$ is reducible if and only if $G$ can be 'separated' as a union of disjoint subgraphs, or graphs joined at a vertex. Also see [MA], $\S 5$, for a discussion of this question.

The conjecture mentioned at the end of the previous section is that $\hat{Y}_{G}$ should determine a mixed-Tate motive (over $\mathbb{Z}$ ). As a consequence, the Grothendieck class $\left[\hat{Y}_{G}\right]$ should belong to the subring $\mathbb{Z}[\mathbb{L}]$ of the Grothendieck ring $K_{0}\left(\operatorname{Var}_{\mathbb{Z}}\right)$; and it would follow that $\hat{Y}_{G}$ is polynomially countable (cf. Definition 2.4. Example 2.5). That is: as it is defined over $\mathbb{Z}, \Psi_{G}$ defines a polynomial in $\mathbb{F}_{q}\left[t_{1}, \ldots, t_{n}\right]$ for all finite fields $\mathbb{F}_{q}$ (here $q$ is of course a prime power); one can then let $N_{q}(G)$ be the number of tuples $\left(t_{1}, \ldots, t_{n}\right)$ in $\mathbb{F}_{q}^{n}$ such that $\Psi_{G}(\bar{t}) \neq 0$ in $\mathbb{F}_{q}$. We have the implications

$$
\hat{Y}_{G} \text { mixed-Tate } \Longrightarrow\left[\hat{Y}_{G}\right] \in \mathbb{Z}[\mathbb{L}] \Longrightarrow N_{q}(G) \text { is a polynomial in } q \text {. }
$$

In this sense $G$ is 'polynomially countable'. The conjecture on the mixed-Tateness of $\hat{Y}_{G}$ can therefore be tested by verifying whether $G$ is polynomially countable. The physicists' evidence mentioned in the previous section motivated the conjecture (attributed to Kontsevich) that all graphs would be polynomially countable.

The history of this problem is interesting. Initial work by combinatorialists R. Stanley ([Sta98]), J. R. Stembridge ([Ste98]), and others appeared to provide very substantial evidence for the conjecture: all graphs with 12 or fewer edges were found to satisfy it. But the conjecture was soon disproved in a remarkable paper by P. Belkale and P. Brosnan ([BB03]), showing that there must be graphs $G$ for which $\left[\hat{Y}_{G}\right]$ is not in the subring $\mathbb{Z}[\mathbb{L}]$ of $K_{0}\left(\operatorname{Var}_{\mathbb{Z}}\right)$. Indeed, Belkale and Brosnan show that classes of graph hypersurfaces generate the Grothendieck ring $K_{0}\left(\operatorname{Var}_{\mathbb{Z}}\right)$, in a sense to which I will come back at the end of 84 . In more recent work by D. Doryn and O. Schnetz ([Dor11], [Sch11]), it has been shown that all graphs with 13 or fewer edges satisfy the conjecture, and specific graphs $G$ with 14 and 16 edges have been found which are not polynomially countable. The examples with 16 edges fail polynomial countability as the corresponding $N_{q}(G)$ can be related to the number of points on a $\mathrm{K} 3$ in $\mathbb{P}^{3}$. The appearance of $\mathrm{K} 3 \mathrm{~s}$ in this context is further studied in the more recent BS12.

The polynomial $\Psi_{G}$ may be written as a determinant of a matrix easily concocted from the graph and recording which edges belong to a fixed basis for $H^{1}(G)$. Thus, graph hypersurfaces may be viewed as specializations of a determinantal hypersurface, and with suitable care one can write Feynman periods as periods of the complement of a determinant hypersurface. After all, the Feynman periods detect one part of the cohomology of $\hat{Y}_{G}$; even if $\hat{Y}_{G}$ is not mixed-Tate, it may be that the part of its cohomology that is responsible for the period is mixed-Tate. Realizing the period as a period over some other potentially simpler locus is a natural approach to proving that it must be a combination of multiple zeta values. This approach is carried out in [AM10, but hits against a wall: even though determinant hypersurfaces are indeed simpler objects than graph hypersurfaces, the intersection of the hypersurface with the domain of integration becomes much harder to grasp. It may be described in terms of intersections of unions of Schubert cells in flag varieties, and these objects are known to be arbitrarily complex (this is one expression of R. Vakil's "Murphy's law", [Vak06]).

In the following $\$ 4$ we will focus on general considerations relating the class $\left[\hat{Y}_{G}\right]$ in $K_{0}$ (Var) with the combinatorics of $G$. We will also have something to say about the other 'generalized Euler characteristic' considered in $\$ 2$, that is, the Chern-Schwartz-MacPherson class of a graph hypersurface $X_{G}$, and an intriguing polynomial invariant which may be 
extracted from it. The very fact that the behavior of this invariant is in many ways analogous to the behavior of the Grothendieck class is interesting; and in any case we view $c_{\mathrm{SM}}\left(X_{G}\right)$ as a means to get global information on the singularities of the hypersurface $X_{G}$. The issue of polynomial countability and its relation to Kontsevich's conjecture was a strong motivation for the work reviewed in $\$$, but it will remain as an undercurrent, while the emphasis will simply be on studying these objects for their own sake.

\section{Grothendieck Classes of Graph hypersurfaces}

4.1. Feynman rules. Recall from $\$ 3.1$ that the action integrals we are interested in take the form (7): $\sum_{\text {graphs } G}\langle G\rangle$, where $\langle G\rangle$ is a contribution due to $G$ and accounting for a symmetry factor. If $G_{i}$ are the connected components of $G$, then

$$
\langle G\rangle=\prod_{i}\left\langle G_{i}\right\rangle / \text { extra symmetries }
$$

The linked cluster theorem states that this 'scattering cross section' can then be decomposed as follows:

$$
\sum_{\text {graphs } G}\langle G\rangle=\exp \left(\sum_{\text {connected graphs } G}\langle G\rangle\right) .
$$

That is, the combinations of the terms due to exponentiating the sum recovers precisely the extra symmetry factors. There is a further reduction: if $G$ is obtained by joining $G_{1}$ and $G_{2}$ by an edge joining a vertex of $G_{1}$ to a vertex of $G_{2}$, then

$$
\langle G\rangle=\text { 'propagator' } \cdot\left\langle G_{1}\right\rangle \cdot\left\langle G_{2}\right\rangle,
$$

where the (inverse) 'propagator' term is a multiplicative factor independent of the graphs, allowing for the 'propagation' of momentum from one graph to the other across the bridge joining the two graphs.

These reductions are parts of the lore of 'Feynman rules', a set of recipes that, given a graph, allow a competent physicist to write down the amplitude due to a graph as an integral by extracting information from the combinatorics of the graph. It is natural to ask

Do invariants such as the Grothendieck or $c_{\text {SM }}$ class of a graph hypersurface satisfy a similar structure?

This appears to be a hard an interesting question. In this subsection we are going to observe that the bare-bone essence of the Feynman rules, i.e., the behavior of the invariants with respect to splitting a graph into connected components, or joining two graphs by a bridge, does indeed apply to our invariants in a form that is very similar to the classical one recalled above. This is straightforward for what concerns the Grothendieck class, but requires some work for the $c_{\mathrm{SM}}$ invariant. In fact, I was rather surprised when it turned out to work 'on the nose' for our $c_{\mathrm{SM}}$ invariant, and I take this instance as good evidence that the invariant is indeed detecting directly some information carried by the combinatorics of the graph.

This material is explored in more detail in AM11a. The reader is warned that the title is more ambitious than the article itself: only the Feynman rules dealing with propagator bridges are really dealt with in this paper. Of course the hope would be that more sophisticated 'algebro-geometric Feynman rules' can be concocted, and eventually allow to write the Grothendieck class or the $c_{\mathrm{SM}}$ class for a graph hypersurface by a straightforward combinatorial recipe. This is a good (and difficult) open question, which is not even touched in AM11a. (One has to start somewhere...)

The following observation is a direct consequence of the definition of graph polynomials: 
Lemma 4.1. Let $G$ be the disjoint union of two graphs $G_{1}, G_{2}$. Then $\Psi_{G}=\left(\Psi_{G_{1}}\right)\left(\Psi_{G_{2}}\right)$.

Therefore, the polynomial of a graph is the product of the polynomials for its connected components. The same happens for components connected by bridges:

Lemma 4.2. Let $G$ be obtained by joining $G_{1}$ and $G_{2}$ by a bridge. Then $\Psi_{G}=\left(\Psi_{G_{1}}\right)\left(\Psi_{G_{2}}\right)$.

The point is that bridges belong to all spanning trees, therefore the corresponding variables do not appear in the graph polynomial.

Example 4.3. Joining two polygons with a bridge:

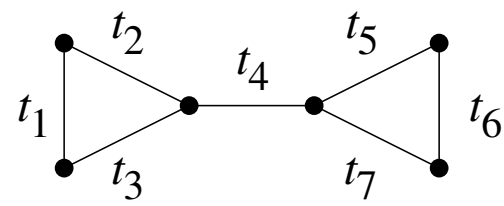

A spanning tree is obtained by joining a spanning tree of the left triangle with a spanning tree of the triangle on the right by the bridge $t_{4}$. It follows easily that the graph polynomial equals $\left(t_{1}+t_{2}+t_{3}\right)\left(t_{5}+t_{6}+t_{7}\right)$. The three graphs

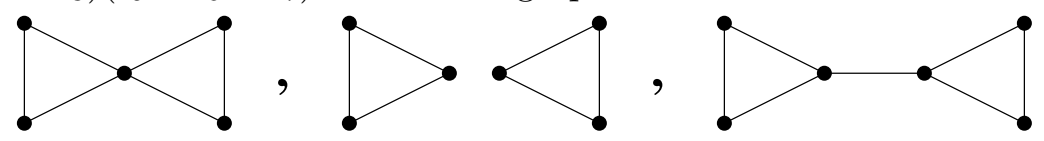

all have the same graph polynomial. But note that the third graph hypersurface lives in a space of higher dimension, because of the extra edge. This fact is responsible for the form of the propagator in our version of the 'Feynman rules'.

Recall that for a graph $G$ on $n$ edges we are denoting by $X_{G}$ the hypersurface $\Psi_{G}\left(t_{1}, \ldots, t_{n}\right)=$ 0 in $\mathbb{P}^{n-1}$, by $\hat{X}_{G}$ the affine cone in $\mathbb{A}^{n}$, and by $\hat{Y}_{G}$ the complement $\mathbb{A}^{n} \backslash \hat{X}_{G}$.

Proposition 4.4. Let $G_{1}, G_{2}$ be graphs.

- If $G=G_{1} \amalg G_{2}$, then $\hat{Y}_{G} \cong \hat{Y}_{G_{1}} \times \hat{Y}_{G_{2}}$.

- If $G$ is obtained by joining $G_{1}$ and $G_{2}$ by a bridge, then $\hat{Y}_{G} \cong \hat{Y}_{G_{1}} \times \hat{Y}_{G_{2}} \times \mathbb{A}^{1}$.

Proof. Let $t_{1}, \ldots, t_{n_{1}}$ be the variables corresponding to the edges of $G_{1}$, and let $u_{1}, \ldots, u_{n_{2}}$ be the variables corresponding to the edges of $G_{2}$. If $G=G_{1} \amalg G_{2}$, then $\Psi_{G}=\Psi_{G_{1}} \Psi_{G_{2}}$ as observed above. A point $\left(t_{1}, \ldots, t_{n_{1}}, u_{1}, \ldots, u_{n_{2}}\right)$ of $\mathbb{A}^{n_{1}+n_{2}}$ is in $\hat{Y}_{G}$ if and only if

$$
\Psi_{G}\left(t_{1}, \ldots, t_{n_{1}}, u_{1}, \ldots, u_{n_{2}}\right) \neq 0 \quad,
$$

if and only if

$$
\Psi_{G_{1}}\left(t_{1}, \ldots, t_{n_{1}}\right) \Psi_{G_{2}}\left(u_{1}, \ldots, u_{n_{2}}\right) \neq 0
$$

if and only if

$$
\Psi_{G_{1}}\left(t_{1}, \ldots, t_{n_{1}}\right) \neq 0 \text { and } \Psi_{G_{2}}\left(u_{1}, \ldots, u_{n_{2}}\right) \neq 0
$$

if and only if $\left(t_{1}, \ldots, t_{n_{1}}\right) \in \hat{Y}_{G_{1}}$ and $\left(u_{1} \ldots, u_{n_{2}}\right) \in \hat{Y}_{2}$. The first assertion follows.

If $G_{1}$ and $G_{2}$ are joined by a bridge, we have precisely the same computation, but in the presence of an extra variable $e$; this accounts for the extra factor of $\mathbb{A}^{1}$, giving the second assertion. (In fact, the second assertion follows from the first.)

Proposition 4.4 says that the very definition of graph hypersurface complement satisfies the 'bare-bone structure' mentioned above. Notice that the extra $\mathbb{A}^{1}$ appearing in the second formula may be interpreted as the graph hypersurface complement for the added bridge joining the two graphs. For us (and for lack of a better term), 'abstract Feynman rules' consist of any assignment from graphs to rings, $G \mapsto U(G)$, such that 
- If $G=G_{1} \amalg G_{2}$ or $G$ is obtained by joining $G_{1}$ and $G_{2}$ at a single vertex, then $U(G)=U\left(G_{1}\right) U\left(G_{2}\right)$.

In particular, if $G$ is obtained by joining $G_{1}$ and $G_{2}$ by a bridge $e$, then $U(G)=U\left(G_{1}\right) U\left(G_{2}\right) U(e)$. This identifies $U(e)$ as the (inverse) 'propagator'.

Abstract Feynman rules are of course a dime for a dozen: for example, it suffices to assign any elements of a ring to connected, 1-PI graphs, and define the invariant on more general graphs by adopting the multiplicativity prescribed by the rules. Or we can simply encode into a 'rule' any feature of the graph that is manifestly additive on disjoint unions. For instance, the following example looks rather useless; but we record it anyway.

Example 4.5. Let $\mathcal{S B}(G) \in \mathbb{Z}$ be 0 if $G$ has edges that are not looping edges, and $(-1)^{n}$ if $G$ has $n$ looping edges and no other edge.

This is trivially an example of 'abstract Feynman rules' in the sense specified above, and it does not look very interesting (yet).

What is more interesting is to identify invariants that are meaningfully and independently defined for all graphs, and verify that these happen to satisfy the multiplicativity prescribed by the rules. We are interested in those invariants that arise from algebro-geometric properties of graph hypersurfaces.

Definition 4.6. Algebro-geometric Feynman rules are abstract Feynman rules which only depend on the affine hypersurface complement $\hat{Y}_{G}$.

It is not difficult to construct 'universal' algebro-geometric Feynman rules, in the form of a Grothendieck ring $\mathscr{F}$ of conical immersed affine varieties, so that algebro-geometric Feynman rules with target a ring $R$ correspond precisely to ring homomorphisms $\mathscr{F} \rightarrow R$. The hypersurface complement $\hat{Y}_{G}$ determines a class in this Grothendieck ring $\mathscr{F}$.

Two examples are discussed below; there are others, and they await further study. In general, it may be not completely trivial to decide whether given Feynman rules are 'algebrogeometric' in the sense of Definition 4.6. For instance, is the assignment $\mathcal{S B}(G)$ of Example 4.5 algebro-geometric?

Also, it may be useful to point out that 'meaningful' algebro-geometric invariants need not be Feynman rules. My favorite example is the Euler characteristic $\chi\left(Y_{G}\right)$ of the complement of the graph hypersurface in projective space: if $G$ is the disjoint union of $G_{1}$ and $G_{2}$, and neither $G_{1}$ nor $G_{2}$ is a forest, then the class $\left[Y_{G}\right]$ is a multiple of ( $\mathbb{L}-1$ ) (exercise!), so $\chi\left(Y_{G}\right)=0$ no matter what $\chi\left(Y_{G_{1}}\right)$ and $\chi\left(Y_{G_{2}}\right)$ may be. By itself, this invariant does not seem to have enough 'structure' to qualify as an example of Feynman rules. By the end of this subsection we will see that the information carried by this invariant can be incorporated into meaningful Feynman rules, but in a rather nontrivial way.

- Motivic algebro-geometric Feynman rules:

Simply send the class of $\hat{Y}_{G}$ in $\mathscr{F}$ to the class $\mathbb{U}(G):=\left[\hat{Y}_{G}\right]$ in the ordinary Grothendieck ring $K_{0}$ (Var). The content of Proposition 4.4 is precisely that this assignment gives 'abstract Feynman rules' in the sense specified above. The 'motivic propagator' is the class $\mathbb{L}=(\mathbb{T}+1)$ of $\mathbb{A}^{1}$. It is a good exercise to establish the effect of some simple graph operations on $\mathbb{U}(G)$; for example, splitting an edge also multiplies $\mathbb{U}(G)$ by the propagator $(\mathbb{T}+1)$, while adding a looping edge multiplies $\mathbb{U}(G)$ by $\mathbb{T}$.

While this example is very straightforward, the reader should note that variations on this theme - such as $\left[\hat{X}_{G}\right],\left[X_{G}\right]$, or the class $\left[Y_{G}\right]$ of the hypersurface complement in projective space, would not work. The class $\left[\hat{Y}_{G}\right]$ of the affine complement is just right.

-Polynomial algebro-geometic Feynman rules: 
A far less straightforward example may be obtained using $c_{\mathrm{SM}}$ classes; the corresponding Feynman rules take values in $A_{*} \mathbb{P}^{n}$, so they may be interpreted as (truncated) polynomials in $\mathbb{Z}[T]$.

Consider any affine cone $\hat{X}$ in $\mathbb{A}^{n}$ as a locally closed subset of the projective space $\mathbb{P}^{n}$ obtained by adding the hyperplane at infinity. The $c_{\mathrm{SM}}$ class of the corresponding indicator function is a combination of classes of projective subspaces, with integer coefficients:

$$
c_{\mathrm{SM}}\left(\mathbb{1}_{\hat{X}}\right)=a_{0}\left[\mathbb{P}^{0}\right]+a_{1}\left[\mathbb{P}^{1}\right]+\cdots+a_{n}\left[\mathbb{P}^{n}\right] .
$$

Associate with $\hat{X}$ the polynomial $F_{\hat{X}}(T):=a_{0}+a_{1} T+\cdots+a_{n} T^{n}$.

Example 4.7. What is $F_{\mathbb{A}^{n}}(T)$ ? Denoting by $H$ the hyperplane class in $\mathbb{P}^{n}$, use (6):

$$
\begin{aligned}
c_{\mathrm{SM}}\left(\mathbb{1}_{\mathbb{A}^{n}}\right) & =c_{\mathrm{SM}}\left(\mathbb{1}_{\mathbb{P}^{n}}-\mathbb{1}_{\mathbb{P}^{n-1}}\right) \\
& =\left((1+H)^{n+1}-(1+H)^{n} H\right) \cap\left[\mathbb{P}^{n}\right]=(1+H)^{n} \cap\left[\mathbb{P}^{n}\right] \\
& =\sum_{i=0}^{n}\left(\begin{array}{c}
n \\
i
\end{array}\right)\left[\mathbb{P}^{i}\right] \leadsto F_{\mathbb{A}^{n}}(T)=\sum_{i=0}^{n}\left(\begin{array}{c}
n \\
i
\end{array}\right) T^{i} .
\end{aligned}
$$

Therefore, $F_{\mathbb{A}^{n}}(T)=(1+T)^{n}$.

Proposition 4.8 (Easy). F defines a group homomorphism $\mathscr{F} \rightarrow \mathbb{Z}[T]$.

Proof. Inclusion-exclusion holds for $c_{\mathrm{SM}}$ classes.

Theorem 4.9 (Not so easy). $F$ defines a ring homomorphism $\mathscr{F} \rightarrow \mathbb{Z}[T]$.

This is somewhat surprising, if only because the identification of $A_{*} \mathbb{P}^{n}$ with truncated polynomials in $\mathbb{Z}[T]$ does not preserve products - the product in $\mathbb{Z}[T]$ does not correspond to the intersection product in $A_{*} \mathbb{P}^{n}$. A priori, there does not seem to be any reasonable sense in which $\left[\mathbb{P}^{i}\right]$ and $\left[\mathbb{P}^{j}\right]$ should 'multiply' to $\left[\mathbb{P}^{i+j}\right]$, especially if $i+j$ exceeds the dimension of the ambient space $\mathbb{P}^{n}$. Proposition 4.9 recovers a way in which this makes sense nevertheless.

Once Theorem 4.9 is established, we can define 'polynomial', or ' $c_{\mathrm{SM}}$ ' Feynman rules by associating with a graph $G$ the polynomial $C_{G}(T):=F_{\hat{Y}_{G}}(T) \in \mathbb{Z}[T]$. The $c_{\text {SM }}$ propagator is the polynomial $C_{\mathbb{A}^{1}}(T)=1+T$. (Notice the formal similarity with the situation with the motivic Feynman rules, once the variable $T$ is 'interpreted' as the class $\mathbb{T}$ of a torus.)

Example 4.10. If $G$ is an $n$-sided polygon, then $C_{G}(T)=T(1+T)^{n-1}$.

To verify this, note that the graph polynomial for an $n$-sided polygon $G$ is simply $t_{1}+$ $\cdots+t_{n}$. Therefore, $\hat{Y}_{G}$ consists of the complement of a hyperplane $\mathbb{A}^{n-1}$ in $\mathbb{A}^{n}$. As verified in Example 4.7, $F_{\mathbb{A}^{r}}(T)=(1+T)^{r}$, and it follows that

$$
C_{G}(T)=F_{\mathbb{A}^{n} \backslash \mathbb{A}^{n-1}}(T)=(1+T)^{n}-(1+T)^{n-1}=(1+T-1)(1+T)^{n-1}=T(1+T)^{n-1}
$$

as claimed.

A number of general properties of $C_{G}(T)$ may be established easily; the reader may take most of these as good exercises in handling the definitions. A much subtler relation with the combinatorics of $G$ will be mentioned below, in $\$ 4.2$.

(1) $C_{G}(T)$ is a monic polynomial, of degree equal to the number $n$ of edges of $G$;

(2) The coefficient of $T^{n-1}$ in $C_{G}(T)$ equals $n-b_{1}(G)$;

(3) If $G$ is a forest with $n$ edges, then $C_{G}(T)=(1+T)^{n}$;

(4) If $G$ is not a forest, then $C_{G}(0)=0$;

(5) $C_{G}^{\prime}(0)$ equals the Euler characteristic of $Y_{G}=\mathbb{P}^{n-1} \backslash X_{G}$; 
(6) If $G^{\prime}$ is obtained from $G$ by attaching an edge or splitting an edge, then $C_{G^{\prime}}(T)=$ $(1+T) C_{G}(T)$

(7) If $G^{\prime}$ is obtained from $G$ by attaching a looping edge, then $C_{G^{\prime}}(T)=T C_{G}(T)$;

(8) If $G$ is not 1-particle irreducible, then $C_{G}(-1)=0$.

These properties often streamline computations. For example, to obtain (again) $C_{G}(T)$ for an $n$-sided polynomial, start from a single vertex and no edges; by (3), the invariant is 1 in this case. By (7), the invariant for a single looping edge is $T$; and then applying (6) $n-1$ times we obtain again that the invariant for an $n$-sided polynomial is $T(1+T)^{n-1}$.

Also: (4) and (5) clarify the Feynman rules role of $\chi\left(Y_{G}\right)$ : If $G_{1}$ and $G_{2}$ are not forests, then $C_{G_{1}}(T)=\chi\left(Y_{G_{1}}\right) T+$ h.o.t and $C_{G_{2}}(T)=\chi\left(Y_{G_{2}}\right) T+$ h.o.t. By Theorem 4.9,

$$
C_{G_{1} \amalg G_{2}}(T)=\chi\left(Y_{G_{1}}\right) \chi\left(Y_{G_{2}}\right) T^{2}+\text { h.o.t } \quad:
$$

this confirms that $\chi\left(Y_{G_{1} \amalg G_{2}}\right)=0$, but tells us that the product $\chi\left(Y_{G_{1}}\right) \chi\left(Y_{G_{2}}\right)$ is not lost in the process: it just appears as the coefficient of $T^{2}$ in the corresponding $c_{\mathrm{SM}}$ Feynman rules.

Incidentally: In all examples I know, $\chi\left(Y_{G}\right)$ is always 0 or \pm 1 . (A large number of computations for small graphs are collected in [Str11].) I have not been able to prove that this holds in general. Based on the above considerations, it is tempting to conjecture the stronger statement that the first nonzero coefficient of $C_{G}(T)$ should always be \pm 1 .

If $G$ is not a forest, then one may verify that $C_{G}(T)$ is the polynomial obtained from $c_{\mathrm{SM}}\left(\mathbb{1}_{\mathbb{P}^{n-1} \backslash X_{G}}\right)$ by replacing $\left[\mathbb{P}^{k}\right]$ with $T^{k+1}$. Naive variations on this recipe, e.g., using $c_{\mathrm{SM}}\left(\mathbb{1}_{X_{G}}\right)$ instead, simply do not work. The computation behind Theorem 4.9 is somewhat delicate.

Example 4.11. Let $G$ be the 3-banana graph:

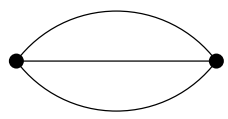

The corresponding graph polynomial is $t_{1} t_{2}+t_{1} t_{3}+t_{2} t_{3}$; therefore, the graph hypersurface $X_{G}$ is a nonsingular conic in $\mathbb{P}^{2}$, hence $c_{\mathrm{SM}}\left(\mathbb{1}_{X_{G}}\right)=2\left[\mathbb{P}^{0}\right]+2\left[\mathbb{P}^{1}\right]$. It follows that $c_{\mathrm{SM}}\left(\mathbb{1}_{\mathbb{P}^{2} \backslash X_{G}}\right)=\left[\mathbb{P}^{0}\right]+\left[\mathbb{P}^{1}\right]+\left[\mathbb{P}^{2}\right]$, and therefore $C_{G}(T)=T\left(1+T+T^{2}\right)$.

For the proof of Theorem 4.9, the reader may consult AM11a]. The surprising role of the product in $\mathbb{Z}[T]$ vis-a-vis multiplication of $c_{\mathrm{SM}}$ classes is clarified to some extent in Alub]. Both Jörg Schürmann and Andrzej Weber have remarked that Theorem 4.9 has an interpretation (and perhaps a more natural proof) in an equivariant setting; see [Web12, Proposition 3.

4.2. Deletion-contraction. An impressive number of important graph invariants turn out to be specializations of the 'Tutte polynomial'. This polynomial encodes the basic recursion in graph theory building up a graph by inserting an edge between two vertices or splitting a vertex into two and joining the two resulting vertices by an edge. It is clear that if one can control the behavior of an invariant under these operations, or equivalently under the operations of removing (' $G \backslash e$ ') or contracting (' $G / e$ ') an edge $e$, then one can in principle recursively compute the invariant for all graphs.

In general, a 'Tutte-Grothendieck invariant' is a function $\tau$ from the set of graphs to $R[\alpha, \beta, \gamma, x, y]$, where $R$ is a ring (usually taken to be $\mathbb{C}$ ) such that

- $\tau(G)=\gamma^{\# \text { vertices }}$ if $G$ has no edges;

- $\tau(G)=x \tau(G \backslash e)$ if $e$ is a bridge;

- $\tau(G)=y \tau(G / e)$ if $e$ is a looping edge; 
- $\tau(G)=\alpha \tau(G / e)+\beta \tau(G \backslash e)$ if $e$ is neither a bridge nor a looping edge.

Examples of Tutte-Grothendieck invariants include the number of spanning trees; the chromatic polynomial; the flow polynomial; the partition function of the Ising model; and many more. Up to a change of variables and minor adjustments, the graph polynomial $\Psi_{G}$ is itself such an invariant.

Fact: Every Tutte-Grothendieck invariant is a specialization of the Tutte polynomial $T_{G}(x, y)$, i.e., the special case $\alpha=\beta=\gamma=1$.

This is surprising at first, but rather easy to prove. The very existence of an invariant such as the Tutte polynomial is in itself somewhat magic. The recursive relations allow us to compute it easily for any small graph, for example:

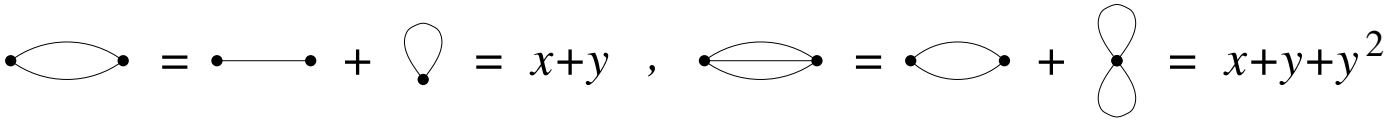

and hence

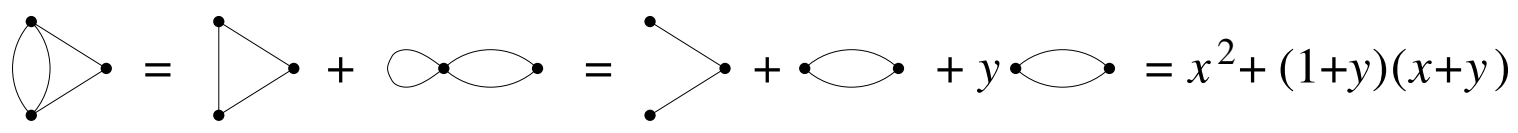

or

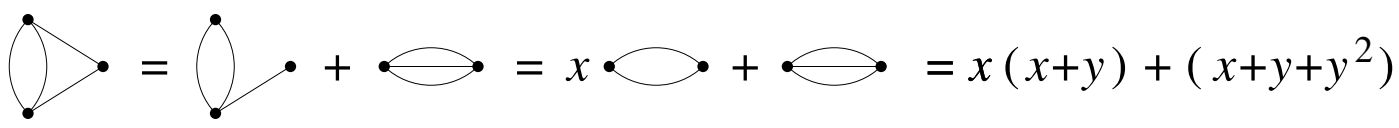

(What is magic is that any choice of sequence of deletions \& contractions necessarily gives the same result.) As an illustration of the specialization result mentioned above, the chromatic polynomial of a graph may be recovered from the Tutte polynomial by setting $x=1-\lambda, y=0$ (and taking care of a sign and a factor of a power of $\lambda$ ). For the graph shown above, this gives $(1-\lambda)^{2}+(1-\lambda)=(1-\lambda)(2-\lambda)$, which equals the chromatic polynomial up to a factor of $\lambda$.

Note that the Tutte polynomial itself is an instance of 'abstract Feynman rules' in the sense of $\$ 4.1$; it is clear from the recursive definition that

$$
T_{G_{1} \cup G_{2}}(x, y)=T_{G_{1}}(x, y) \cdot T_{G_{2}}(x, y)
$$

if $G_{1}$ and $G_{2}$ are have at most one vertex in common; the propagator factor is $x$. Also, the recursive definition of $T_{G}(x, y)$ implies a formula for doubling a single edge in a graph: if $e$ is an edge of $G$, denote by $G_{2 e}$ the graph obtained by inserting an edge parallel to $e$ in $G$; then

$$
\begin{aligned}
T_{G_{2 e}}(x, y) & =T_{G}(x, y)+y T_{G / e}(x, y) \\
& =T_{G \backslash e}(x, y)+(y+1) T_{G / e}(x, y)
\end{aligned}
$$

if $e$ is neither a bridge nor a looping edge (this is a good exercise for the reader). For example, this formula produces the Tutte polynomial for the graph (shown above) obtained by doubling an edge of a triangle from the polynomial $x^{2}+x+y$ for the triangle and the polynomial $x+y$ for a 2-banana. A little care produces multiple edge formulas, and it is easy to put these together into a generating function,

$$
\sum_{m \geq 0} T_{G_{m e}}(x, y) \frac{s^{m}}{m !}=e^{s}\left(T_{G \backslash e}(x, y)+\frac{e^{(y-1) s}-1}{y-1} T_{G / e}(x, y)\right),
$$



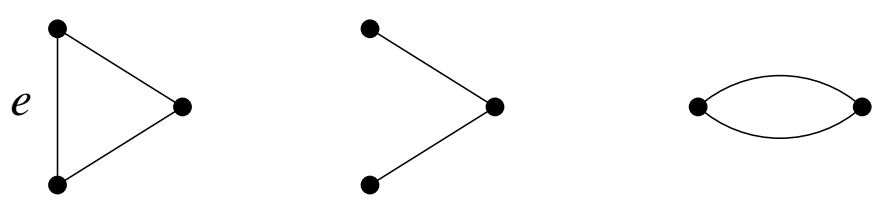

Figure 1. $G, G \backslash e, G / e$.

again under the assumption that $e$ is neither a bridge nor a looping edge. The details of these formulas are not important, but the reader is invited to keep in mind their general shape.

At this point it is natural to ask

Are invariants such as the motivic and $c_{\mathrm{SM}}$ Feynman rules discussed in 4.1

Tutte-Grothendieck invariants?

That is: are these Feynman rules specializations of the Tutte polynomial? The quick answer is 'no', as simple examples show. There is a more cogent reason why the answer must be no: if the motivic Feynman rule were a Tutte-Grothendieck invariant, then the Grothendieck class of the complement $\hat{Y}_{G}$ would recursively be in $\mathbb{Z}[\mathbb{L}]$, while the result of Belkale and Brosnan ([BB03]; see the discussion in \$3.2) guarantees that this is not the case. On the other hand, the behavior under the deletion-contraction of these invariants can be understood to some extent, and one can verify that part of the structure implied by the axioms listed above does hold for the algebro-geometric Feynman rules encountered in 4.1 specifically, we can establish doubling edge formulas which have the same flavor, if not exact shape, as the one shown above for the Tutte polynomial. Details for this material may be found in AM11b] and Alua].

As usual, the result is more straightforward for the motivic Feynman rules. Recall from $\$ 4.1$ that $\mathbb{U}(G)$ denotes the class $\left[\hat{Y}_{G}\right]$ in $K_{0}$ (Var).

Theorem 4.12. Let e be an edge of $G$, and assume $e$ is neither a bridge nor a looping edge. Then

- $\mathbb{U}(G)=\mathbb{L} \cdot\left[\hat{Y}_{G \backslash e} \cup \hat{Y}_{G / e}\right]-\mathbb{U}(G \backslash e)$,

- $\mathbb{U}\left(G_{2 e}\right)=(\mathbb{T}-1) \cdot \mathbb{U}(G)+\mathbb{T} \cdot \mathbb{U}(G \backslash e)+(\mathbb{T}+1) \cdot \mathbb{U}(G / e)$.

The first formula is a version of a deletion-contraction relation for motivic Feynman rules. In one form or another, this formula has been noticed by anyone working on polynomial countability of graph hypersurfaces, starting at least as early as Ste98. The 'problem' with it is that it is not purely combinatorial: the term in the middle involves a locus, $\hat{Y}_{G \backslash e} \cup \hat{Y}_{G / e}$, which does not seem accessible directly in terms of Feynman rules. In a sense it cannot be accessible, as mentioned earlier, by the result of [BB03].

The second formula is combinatorial, in the sense that it only involves motivic Feynman rules for graphs obtained from the given one by combinatorial operations. For example, from the knowledge of $\mathbb{U}(G)$ for the graphs in Figure 1, to wit $\mathbb{L}^{3}-\mathbb{L}^{2}=\mathbb{T}(\mathbb{T}+1)^{2},(\mathbb{T}+1)^{2}$, $\mathbb{T}(\mathbb{T}+1)$ (exercise!), we get that the motivic class for the triangle with doubled edge is

$$
(\mathbb{T}-1) \mathbb{T}(\mathbb{T}+1)^{2}+\mathbb{T}(\mathbb{T}+1)^{2}+(\mathbb{T}+1) \mathbb{T}(\mathbb{T}+1)=\mathbb{T}(\mathbb{T}+1)^{3}
$$

This is as it should be, as the graph may also be obtained by splitting an edge in a 3-banana, and the class of a 3 -banana is $\mathbb{T}(\mathbb{T}+1)^{2}$. A complete computation of the Grothendieck class for all banana graphs may also be found in [MA]. 
In terms of double-edge formulas, the situation is entirely similar to the situation for the Tutte polynomial. As in that case, we get as a formal consequence a generating function for the result of 'multiplying' (bananifying?) the edge $e$ :

$$
\sum_{m \geq 0} \mathbb{U}\left(G_{m e}\right) \frac{s^{m}}{m !}=\frac{e^{\mathbb{T} s}-e^{-s}}{\mathbb{T}+1} \mathbb{U}(G)+\frac{e^{\mathbb{T} s}+\mathbb{T} e^{-s}}{\mathbb{T}+1} \mathbb{U}(G \backslash e)+\left(s e^{\mathbb{T} s}-\frac{e^{\mathbb{T} s}-e^{-s}}{\mathbb{T}+1}\right) \mathbb{U}(G / e) .
$$

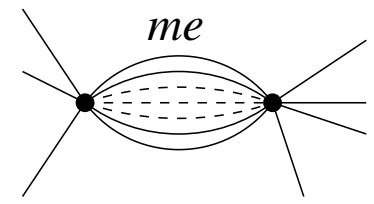

Our conclusion is that although deletion-contraction does not hold for the motivic Feynman rules, some further consequences do hold 'as if' motivic Feynman rules were Tutte invariants.

Remark 4.13. (i) The second formula in Theorem 4.12 implies that if $G, G \backslash e, G / e$ are polynomially countable, then so are all $G_{m e}$.

(ii) The coefficients appearing in the formula for the generating function are functions used in defining Hirzebruch's $T_{y}$ genus, cf. $\S 11$ of Chapter III of [Hir95].

(iii) The generating function for $\mathbb{U}\left(G_{m e}\right)$ implies that

$$
\chi\left(Y_{G_{m e}}\right)=(-1)^{m-1}\left(\chi\left(Y_{G}\right)-\chi\left(Y_{G / e}\right)\right),
$$

for $m \geq 2$, still under the assumption that $e$ is neither a bridge nor a looping edge in $G$, and further that $G \backslash e$ is not a forest. As mentioned in $\$ 4.1, \chi\left(Y_{G}\right)$ is always 0 or \pm 1 in all examples I have been able to compute. If this were necessarily the case, then this formula would imply that $\chi\left(Y_{G}\right)$ and $\chi\left(Y_{G / e}\right)$ cannot be both nonzero and opposite in sign under these assumptions.

(iv) For $G=$ a 2-banana (so that $\mathbb{U}(G)=\mathbb{T}(\mathbb{T}+1), \mathbb{U}(G \backslash e)=\mathbb{T}+1$, and $\mathbb{U}(G / e)=\mathbb{T}$ ), we obtain a generating function for the class of $G_{m e}=$ the $(m+1)$-th banana. This recovers the result of the direct computations in $\mathrm{AM} 09$ and $\mathrm{MA}$.

Proof of Theorem 4.12. The first formula is based on a very useful observation regarding graph polynomials: if $e$ is neither a bridge nor a looping edge, then there is a one-to-one correspondence between the maximal forests of $G$ not containing $e$ and the maximal forests of $G \backslash e$, and a one-to-one correspondence between the maximal forests of $G$ containing $e$ and the maximal forests of $G / e$. Denoting by $t_{e}$ the variable corresponding to $e$, this shows that

$$
\Psi_{G}=t_{e} \Psi_{G \backslash e}+\Psi_{G / e} .
$$

Now let $\underline{t} \in \mathbb{A}^{n}, n=$ number of edges of $G$. We have

$$
\underline{t} \in Y_{G} \Longleftrightarrow \Psi_{G}(\underline{t}) \neq 0 \Longleftrightarrow \Psi_{G / e}\left(\underline{t}^{\prime}\right) \neq t_{e} \Psi_{G \backslash e}\left(\underline{t}^{\prime}\right) \quad,
$$

where $\underline{t}^{\prime}$ denotes omission of $t_{e}$. Now a simple case-by-case analysis proves the first formula in Theorem 4.12. For example, the case in which $\underline{t}^{\prime} \notin Y_{G \backslash e}$ and $\underline{t}^{\prime} \in Y_{G / e}$ accounts for a term

$$
\mathbb{L} \cdot\left[\left(Y_{G \backslash e} \cup Y_{G / e}\right) \backslash Y_{G \backslash e}\right],
$$

with the $\mathbb{L}$ factor due to the freely varying $t_{e}$. Adding the terms obtained from the different possibilities gives the right-hand side of the first formula.

For the second part, assume that $e$ and $e^{\prime}$ are parallel edges in $G_{2 e}$. Then sorting the maximal forests of $G_{2 e}$ according to whether they contain $e$ or $e^{\prime}$ (they cannot contain both) 
gives

$$
\Psi_{G_{2 e}}=t_{e} t_{e^{\prime}} \Psi_{G \backslash e}+\left(t_{e}+t_{e^{\prime}}\right) \Psi_{G / e} .
$$

This formula can be used to obtain a relation between the classes $\left[Y_{G_{2 e} / e} \cup Y_{G_{2 e} \backslash e}\right]$ and $\left[Y_{G / e} \cup Y_{G \backslash e}\right]$. A serendipitous cancellation gets rid of these 'noncombinatorial' terms, and the second formula follows. The reader is invited to provide details for this argument, which may be found in AM11b.

There is an analogous story for $c_{\mathrm{SM}}$ Feynman rules $C_{G}(T)$, but it is more complex, and the proofs are substantially harder. There is a deletion-contraction formula:

Theorem 4.14. Let $e$ be an edge of $G$, and assume $e$ is neither a bridge nor a looping edge, and satisfies two additional technical conditions. Then

$$
C_{G}(T)=C_{G \backslash e, G / e}(T)+(T-1) C_{G \backslash e}(T) .
$$

Here, $C_{G \backslash e, G / e}(T)$ is a 'non-combinatorial term' obtained from the union $\hat{Y}_{G \backslash e} \cup \hat{Y}_{G / e}$ by the same process (described in $\$ 4.1$ yielding $C_{G}(T)$ from $\hat{Y}_{G}$. The two additional technical conditions are somewhat mysterious, and I address the reader to [Alua, $\S 2$ for a thorough discussion. For example, $\Psi_{G / e}(T)$ is required to belong to the Jacobian ideal of $\Psi_{G \backslash e}$. It is a little surprising that this condition holds for as many graphs as it appears to hold; the smallest example of a graph that does not satisfy it is

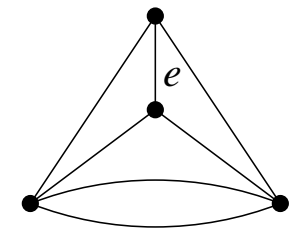

with respect to the vertical edge $e$. (The second condition is even more technical.) It would be interesting to provide combinatorial interpretations of these algebraic conditions. If the conditions are satisfied, we obtain a 'combinatorial' double-edge formula for $c_{\mathrm{SM}}$ Feynman rules:

Theorem 4.15. Under the same hypotheses of Theorem 4.14.

$$
C_{G_{2 e}}(T)=(2 T-1) C_{G}(T)-T(T-1) C_{G \backslash e}(T)+C_{G / e}(T) .
$$

The technical conditions are satisfied for sides of a triangle, so knowledge of $c_{\text {SM }}$ Feynman rules for the graphs in Figure 1, that is,

$$
T(1+T)^{2} \quad, \quad(1+T)^{2} \quad, \quad T(1+T)
$$

(obtained by applying the properties of $c_{\mathrm{SM}}$ Feynman rules listed in 4.1 gives

$$
C_{G}(T)=(2 T-1) T(1+T)^{2}-T(T-1)(1+T)^{2}+T(1+T)=T(1+T)\left(1+T+T^{2}\right)
$$

for the triangle with a doubled edge. This is as it should be, since this graph may be obtained by splitting an edge of a 3-banana, and $C_{3 \text {-banana }}(T)=T\left(1+T+T^{2}\right)$ (Example 4.11).

By a remarkable stroke of luck, the conditions are automatically satisfied if $e$ is a multiple edge of $G$, that is, if its endpoints are adjacent in $G \backslash e$. Thus, the formula given in Theorem 4.15 holds in this case. This is enough to trigger induction, and we get the relation

$$
C_{G_{(m+3) e}}(T)=(3 T-1) C_{G_{(m+2) e}}(T)-\left(3 T^{2}-2 T\right) C_{G_{(m+1) e}}(T)+\left(T^{3}-T^{2}\right) C_{G_{m e}}(T) .
$$

for $m \geq 1$. Note the differentiation relations among the coefficients; I was not able to explain this remarkable feature of the formula to my satisfaction. 
The reader may enjoy the task of obtaining a generating function from Theorem 4.15 .

$$
\begin{aligned}
\sum_{m \geq 0} C_{\Gamma_{(m+1) e}}(t) \frac{s^{m}}{m !} & =\left(e^{t s}-e^{(t-1) s}\right) C_{\Gamma_{2 e}}(t) \\
& -\left((t-1) e^{t s}-t e^{(t-1) s}\right) C_{\Gamma}(t)+t\left((s-1) e^{t s}+e^{(t-1) s}\right) C_{\Gamma / e}(t) .
\end{aligned}
$$

A proof of Theorem 4.14 is obtained by interpreting the basic relation (9):

$$
\Psi_{G}=t_{e} \Psi_{G \backslash e}+\Psi_{G / e}
$$

(for $e$ neither a bridge nor a looping edge in $G$ ) in terms of blow-ups. Assume $G$ has $n$ edges and (for simplicity) that $X_{G}$ is irreducible. Let $p \in \mathbb{P}^{n-1}$ be the point for which all coordinates except $t_{e}$ are set to 0 . Then one may use (9) to verify that the blow-up $B \ell_{p} X_{G}$ of $X_{G}$ at $p$ is isomorphic to the blow-up of $\mathbb{P}^{n-2}$ along $X_{G \backslash e} \cap X_{G / e}$. (Note that it follows that irreducible graph hypersurfaces are rational.) The basic functoriality property of $c_{\text {SM }}$ classes may then be used to relate the various classes, and yields Theorem 4.14. This computation requires that the exceptional divisor of the blow-up of $\mathbb{P}^{n-1}$ at $p$ be sufficiently transversal to the proper transform of $X_{G}$; this in turn translates into the technical conditions mentioned in the statement of Theorem 4.14 .

Again, our conclusion is that while these algebro-geometric Feynman rules are not TutteGrothendieck invariants, they share some basic element of the structure of these invariants, which triggers multiple edge formulas. It would be worth formalizing this observation in more technical terms.

4.3. Stable birational equivalence. One remarkable application of the deletion-contraction relation in Theorem 4.12 is the complete determination of the stable birational equivalence class of graph hypersurfaces.

Definition 4.16. Two irreducible, nonsingular, compact complex algebraic varieties $X, Y$ are stably birationally equivalent if $X \times \mathbb{P}^{\ell}$ and $Y \times \mathbb{P}^{m}$ are birationally equivalent for some $\ell, m$.

Recall ( 8 2.1) that $X$ and $Y$ are 'birationally equivalent' if there are dense open sets $U$ in $X$ and $V$ in $Y$ such that $U$ and $V$ are isomorphic. Stable birational equivalence is a weakening of this condition. If $X_{1}$ is (stably) birationally equivalent to $X_{2}$ and $Y_{1}$ is (stably) birationally equivalent to $Y_{2}$, then $X_{1} \times Y_{1}$ is (stably) birationally equivalent to $X_{2} \times Y_{2}$.

It is not hard to show that two nonsingular compact curves are birationally equivalent if and only if they are in fact isomorphic. In higher dimension, birational equivalence is much more interesting: for example, a variety is birationally equivalent to any of its blow-ups; it follows that, in dimension $\geq 2$, every variety is birationally equivalent to infinitely many varieties that are mutually non-isomorphic. In fact, blow-ups are the prime example of birational equivalence, in the sense that if $X$ and $Y$ are birationally equivalent, then there exists a sequence of blow-ups and blow-downs at smooth centers which transforms $X$ into $Y$. This is the content of the 'weak factorization theorem' of AKMW02, a fundamental tool in the study of birational equivalence.

A variety is rational if it is birationally equivalent to projective space.

We could adopt the definition of stable birational equivalence given above also for singular, or noncompact varieties; thus, $\mathbb{A}^{1}$ or a nodal cubic plane curve would be examples of stably rational varieties. In fact, since (by Hironaka) every complex algebraic variety has a nonsingular compact model, every variety would belong to a well-defined stable birational equivalence class, in this naive sense. 
However, something much more interesting can be done. Larsen and Lunts define ([LL03]) a ring of stable birational equivalence classes as follows:

- Let $S B$ be the monoid of stable birational equivalence classes of smooth irreducible compact varieties, defining the class of $X \times Y$ as the product of the classes of $X$ and $Y$ (this is well-defined, as follows from the facts mentioned above);

- Then let $\mathbb{Z}[S B]$ be the corresponding monoid ring.

Denote by $[X]_{S B}$ the class of $X$ in $\mathbb{Z}[S B]$. Thus, elements of $\mathbb{Z}[S B]$ may be written as formal integer linear combinations of elements $[X]_{S B}$; if $X$ is smooth irreducible compact and rational, then $[X]_{S B}=1$.

Now the main remark is that every variety (possibly singular, noncompact) determines a class in $\mathbb{Z}[S B]$; but for $X$ singular or noncompact, the class of $X$ in $\mathbb{Z}[S B]$ is not necessarily its naive 'stable birational equivalence class' mentioned above. Instead, one can determine the class of $X$ in $\mathbb{Z}[S B]$ by (for example) obtaining $X$ from smooth irreducible compact varieties by ordinary set-theoretic operations, and mapping the result to $\mathbb{Z}[S B]$ by interpreting disjoint union as addition. Of course one must show that this leads to a well-defined class; see Theorem 4.19 below.

Example 4.17. The class of $\mathbb{A}^{1}$ in $\mathbb{Z}[S B]$ is 0 . Indeed, we can view $\mathbb{A}^{1}$ as $\mathbb{P}^{1} \backslash \mathbb{P}^{0}$, hence map it to $\left[\mathbb{P}^{1}\right]_{S B}-\left[\mathbb{P}^{0}\right]_{S B}=1-1=0$.

The class of a nodal plane cubic is also 0: the complement of the node may be realized as the complement of 2 points in $\mathbb{P}^{1}$, so it has class $\left[\mathbb{P}^{1}\right]_{S B}-2\left[\mathbb{P}^{0}\right]_{S B}=-1$; adding the node back gives 0 .

The class of a cuspidal cubic in $\mathbb{Z}[S B]$ is 1 : the complement of the cusp is isomorphic to $\mathbb{A}^{1}$, so has class 0 as seen above; and adding back the cusp gives 1 .

These three examples show that the class of a singular or noncompact rational variety may not be 1 in $\mathbb{Z}[S B]$, but sometime is - this is a rather sophisticated statement about the type of singularities of the variety. Our graph hypersurfaces $X_{G}$ in projective space are usually singular and (when irreducible) rational; it is natural to pose the problem:

Compute the class of $X_{G}$ in the Larsen-Lunts ring $\mathbb{Z}[S B]$ of stable birational equivalence classes.

Theorem 4.18. Let $G$ be a graph that is not a forest, and with at least one non-looping edge. Then the projective graph hypersurface $X_{G}$ has class 1 in $\mathbb{Z}[S B]$.

This is Corollary 3.3 in [AM11c] its proof is very easy modulo [LL03, and is reviewed below. As Example 4.17 hopefully illustrates, the fact that $X_{G}$ is rational (when irreducible) does not suffice to imply that its class in $\mathbb{Z}[S B]$ is 1 , because $X_{G}$ is singular. The fact expressed by Theorem 4.18 is not obvious, even if its proof is very straightforward modulo well-known facts.

Theorem 4.18 follows from the deletion-contraction relation in Theorem 4.12 and from the following key description of the ring $\mathbb{Z}[S B]$ :

Theorem 4.19 (Larsen \& Lunts: [LL03], Theorem 2.3, Proposition 2.7). The assignment $X \mapsto[X]_{S B}$ for $X$ smooth, irreducible, complete descends to an onto ring homomorphism $K_{0}(\operatorname{Var}) \rightarrow \mathbb{Z}[S B]$, whose kernel is the ideal $(\mathbb{L})$.

This result leads to the description given above for the construction of the class in $\mathbb{Z}[S B]$ determined by a possibly singular or noncompact variety: every such variety has a class in $K_{0}$ (Var) which may be expressed in terms of irreducible nonsingular compact varieties, by means of basic set-theoretic operations. 
One way to prove Theorem 4.19 is to show that stable birational equivalence is compatible with Bittner's relations (cf. [LL03, Remark 2.4). This is because if $B$ is a nonsingular compact variety and $E$ is the total space of a projective bundle over $B$, then $B$ and $E$ are stably birationally equivalent: indeed, $E$ is birationally equivalent to $B \times \mathbb{P}^{r}$ for some $r$. It follows that if $X$ is nonsingular and compact, and $B \subset X$ is a nonsingular closed subvariety, then

$$
\left[B \ell_{B} X\right]_{S B}-[E]_{S B}=[X]_{S B}-[B]_{S B}
$$

where $B \ell_{B} X$ is the blow-up of $X$ along $B$, and $E$ is the exceptional divisor. This shows that there is a homomorphism $K_{0}$ (Var) $\rightarrow \mathbb{Z}[S B]$ as claimed, and this homomorphism is onto since classes of nonsingular compact irreducible varieties generate $K_{0}$ (Var). It is clear that $(\mathbb{L})$ is in the kernel of this homomorphism (exercise!); the opposite inclusion may be obtained as a consequence of the weak factorization theorem of [AKMW02]: since birational isomorphisms may be decomposed in terms of blow-ups at nonsingular centers, the key point is that $\left[B \ell_{B} X\right] \equiv[X]$ modulo $(\mathbb{L})$ in $K_{0}$ (Var).

By Theorem 4.19, we can define 'stable birational Feynman rules' by taking the stable birational equivalence class of the motivic Feynman rules $\mathbb{U}(G)$ of 4.1 modulo $\mathbb{L}$ : this defines a homomorphism $\mathscr{F} \rightarrow \mathbb{Z}[S B]$ from the ring of universal algebro-geometric Feynman rules to the Larsen-Lunts ring. As we will see in a moment, this is essentially the same as my prime example (Example 4.5) of not-so-interesting Feynman rules! (But this shows that that example $i s$ algebro-geometric.)

Proof of Theorem 4.18. By Theorem 4.19, it suffices to compute the class of $X_{G}$ modulo $\mathbb{L}$. Equivalently, we can compute the class of $\left[\hat{Y}_{G}\right]=\mathbb{U}(G)$ modulo $\mathbb{L}$. Reading the deletioncontraction formula in Theorem 4.12 modulo $\mathbb{L}$ gives

$$
\mathbb{U}(G) \equiv-\mathbb{U}(G \backslash e) \quad \bmod (\mathbb{L})
$$

if $e$ is neither a bridge nor a looping edge in $G$. If $e$ is a looping edge, then

$$
\mathbb{U}(G)=(\mathbb{L}-1) \mathbb{U}(G \backslash e) \equiv-\mathbb{U}(G \backslash e) \quad \bmod (\mathbb{L}) ;
$$

and if $e$ is a bridge, then

$$
\mathbb{U}(G)=\mathbb{L} \mathbb{U}(G \backslash e) \equiv 0 \quad \bmod (\mathbb{L})
$$

(as we saw in 4.1 . These recursions reduce the computation of $\mathbb{U}(G) \bmod \mathbb{L}$ to the case in which $G$ has no edges at all; and if $G$ had a non-looping edge, at some stage in the reduction process that edge will become a bridge, killing the class modulo $\mathbb{L}$. It follows that

$$
\mathbb{U}(G)=\left\{\begin{array}{lll}
0 & \bmod (\mathbb{L}) & \text { if } G \text { has edges that are not looping edges } \\
(-1)^{n} & \bmod (\mathbb{L}) & \text { if } G \text { has } n \text { looping edges and no other edge. }
\end{array}\right.
$$

This is of course nothing but the assignment $\mathcal{S B}(G)$ of Example 4.5, followed by the inclusion $\mathbb{Z} \rightarrow \mathbb{Z}[S B]$

The statement for $X_{G}$ given in Theorem 4.18 is an easy consequence of this, left to the reader.

The observation that $\left[\hat{Y}_{G}\right]$ is a multiple of $\mathbb{L}$ as soon as $G$ has a non-looping edge may be strengthened: Lemma 15 in BS12] shows that $\left[\hat{Y}_{G}\right]$ is a multiple of $\mathbb{L}^{2}$ for all 'physically significant' graphs.

An interesting consequence of Theorem 4.18 is that the classes of graph hypersurfaces in $\mathbb{Z}[S B]$ span $\mathbb{Z}$, and this is a rather small part of the ring of stable birational equivalence classes. By Theorem 4.19, classes of graph hypersurfaces span a small part of $K_{0}$ (Var): any naive motive $M$ that can be written as a combination of graph hypersurfaces must be of 
the form $c+\mathbb{L} \cdot M^{\prime}$ for some $c \in \mathbb{Z}$ and $M^{\prime} \in K_{0}$ (Var). This is very special-for example, it implies that the Hodge numbers $h^{p, q}$ of $M$ must be zero if $p=0, q>0$ or $p<0, q=0$.

This may not seem very surprising: as a consequence of Theorem 4.19 ([LL03, Corollary 2.6) every class of $K_{0}$ (Var) may be written as a combination of classes of smooth complete varieties that are uniquely determined up to stable rational equivalence; thus one may suspect that if the class of a variety is a combination of classes of graph hypersurfaces, then the variety must itself be stably birationally equivalent to a graph hypersurface. However, I do not know if this is true: note again that graph hypersurfaces are singular in general, so the quoted result does not apply. But Theorem 4.18 does apply and shows that these classes are very special in any case. The conclusion we draw from Theorem 4.18 is precisely that classes of graph hypersurfaces are 'very special' in $K_{0}$ (Var).

This should be contrasted with the result of Belkale and Brosnan disproving Kontsevich's conjecture, mentioned in $\$ 3.2$, whose essence is that classes of graph hypersurfaces are as general as possible: according to [BB03, p. 149, the complements $\hat{Y}_{G}$ are 'from the standpoint of their zeta functions, the most general schemes possible'. The contrast is due to the fact that Belkale and Brosnan work in a localization of $K_{0}\left(\operatorname{Var}_{\mathbb{Z}}\right)$, in which both $\mathbb{L}^{n}-1(n>0)$ and $\mathbb{L}$ are invertible: in BB03 it is shown that classes of graph hypersurfaces generate this localization over the localization of $\mathbb{Z}[\mathbb{L}]$. It follows from Theorem 4.18 that the localization at $\mathbb{L}$ is necessary for this result to hold: classes of graph hypersurfaces do not generate the localization of $K_{0}\left(\operatorname{Var}_{\mathbb{Z}}\right)$ if only $\mathbb{L}^{n}-1$ are inverted. (Indeed, these elements are already invertible modulo $\mathbb{L}$.)

Question: Do graph hypersurfaces generate $K\left(\operatorname{Var}_{\mathbb{Z}}\right)_{\mathbb{L}}$ over $\mathbb{Z}[\mathbb{L}]_{\mathbb{L}}$ ?

\section{REFERENCES}

[AB08] Paolo Aluffi and Jean-Paul Brasselet. Une nouvelle preuve de la concordance des classes définies par M.-H. Schwartz et par R. MacPherson. Bull. Soc. Math. France, 136(2):159-166, 2008.

[AKMW02] Dan Abramovich, Kalle Karu, Kenji Matsuki, and Jarosław Włodarczyk. Torification and factorization of birational maps. J. Amer. Math. Soc., 15(3):531-572, 2002.

[Alua] Paolo Aluffi. Chern classes of graph hypersurfaces and deletion-contraction. arXiv:1106.1447.

[Alub] Paolo Aluffi. Euler characteristics of general linear sections and polynomial Chern classes. arXiv:1207.6638.

[Alu06] Paolo Aluffi. Classes de Chern des variétés singulières, revisitées. C. R. Math. Acad. Sci. Paris, 342(6):405-410, 2006.

[Alu12] Paolo Aluffi. Grothendieck classes and Chern classes of hyperplane arrangements. Int. Math. Res. Not., 2012.

[AM09] Paolo Aluffi and Matilde Marcolli. Feynman motives of banana graphs. Commun. Number Theory Phys., 3(1):1-57, 2009.

[AM10] Paolo Aluffi and Matilde Marcolli. Parametric Feynman integrals and determinant hypersurfaces. Adv. Theor. Math. Phys., 14(3):911-963, 2010.

[AM11a] Paolo Aluffi and Matilde Marcolli. Algebro-geometric Feynman rules. Int. J. Geom. Methods Mod. Phys., 8(1):203-237, 2011.

[AM11b] Paolo Aluffi and Matilde Marcolli. Feynman motives and deletion-contraction relations. In Topology of algebraic varieties and singularities, volume 538 of Contemp. Math., pages 21-64. Amer. Math. Soc., Providence, RI, 2011.

[AM11c] Paolo Aluffi and Matilde Marcolli. Graph hypersurfaces and a dichotomy in the Grothendieck ring. Lett. Math. Phys., 95(3):223-232, 2011.

[BB03] Prakash Belkale and Patrick Brosnan. Matroids, motives, and a conjecture of Kontsevich. Duke Math. J., 116(1):147-188, 2003.

[BEK06] Spencer Bloch, Hélène Esnault, and Dirk Kreimer. On motives associated to graph polynomials. Comm. Math. Phys., 267(1):181-225, 2006.

[Bit04] Franziska Bittner. The universal Euler characteristic for varieties of characteristic zero. Compos. Math., 140(4):1011-1032, 2004. 
[BK97] D. J. Broadhurst and D. Kreimer. Association of multiple zeta values with positive knots via Feynman diagrams up to 9 loops. Phys. Lett. B, 393(3-4):403-412, 1997.

[BK08] Spencer Bloch and Dirk Kreimer. Mixed Hodge structures and renormalization in physics. Commun. Number Theory Phys., 2(4):637-718, 2008.

[Bro] Francis Brown. On the periods of some Feynman integrals. arXiv:0910.0114.

[Bro09] Francis Brown. The massless higher-loop two-point function. Comm. Math. Phys., 287(3):925958, 2009.

[Bro12] Francis Brown. Mixed Tate motives over $\mathbb{Z}$. Ann. of Math. (2), 175(2):949-976, 2012.

[BS81] Jean-Paul Brasselet and Marie-Hélène Schwartz. Sur les classes de Chern d'un ensemble analytique complexe. In Caractéristique d'Euler-Poincaré, volume 83 of Astérisque, pages 93-147. Soc. Math. France, Paris, 1981.

[BS12] Francis Brown and Oliver Schnetz. A K3 in $\phi^{4}$. Duke Math. J., 161(10):1817-1862, 2012.

[BSY10] Jean-Paul Brasselet, Jörg Schürmann, and Shoji Yokura. Hirzebruch classes and motivic Chern classes for singular spaces. J. Topol. Anal., 2(1):1-55, 2010.

[CM] Ozgur Ceyhan and Matilde Marcolli. Feynman integrals and periods in configuration spaces. arXiv:1207.3544.

[Dor11] Dzmitry Doryn. On one example and one counterexample in counting rational points on graph hypersurfaces. Lett. Math. Phys., 97(3):303-315, 2011.

[Ful84a] William Fulton. Intersection theory. Springer-Verlag, Berlin, 1984.

[Ful84b] William Fulton. Introduction to intersection theory in algebraic geometry, volume 54 of $C B M S$ Regional Conference Series in Mathematics. Published for the Conference Board of the Mathematical Sciences, Washington, DC, 1984.

[Ful93] William Fulton. Introduction to toric varieties, volume 131 of Annals of Mathematics Studies. Princeton University Press, Princeton, NJ, 1993. The William H. Roever Lectures in Geometry.

[GNA02] Francisco Guillén and Vicente Navarro Aznar. Un critère d'extension des foncteurs définis sur les schémas lisses. Publ. Math. Inst. Hautes Études Sci., (95):1-91, 2002.

[GS96] H. Gillet and C. Soulé. Descent, motives and K-theory. J. Reine Angew. Math., 478:127-176, 1996.

[Hir64] Heisuke Hironaka. Resolution of singularities of an algebraic variety over a field of characteristic zero. I, II. Ann. of Math. (2) 79 (1964), 109-203; ibid. (2), 79:205-326, 1964.

[Hir75] Heisuke Hironaka. Triangulations of algebraic sets. In Algebraic geometry (Proc. Sympos. Pure Math., Vol. 29, Humboldt State Univ., Arcata, Calif., 1974), pages 165-185. Amer. Math. Soc., Providence, R.I., 1975.

[Hir95] Friedrich Hirzebruch. Topological methods in algebraic geometry. Classics in Mathematics. Springer-Verlag, Berlin, 1995. Translated from the German and Appendix One by R. L. E. Schwarzenberger, With a preface to the third English edition by the author and Schwarzenberger, Appendix Two by A. Borel, Reprint of the 1978 edition.

[Ken90] Gary Kennedy. MacPherson's Chern classes of singular algebraic varieties. Comm. Algebra, 18(9):2821-2839, 1990.

[Kwi92] Michał Kwieciński. Formule du produit pour les classes caractéristiques de Chern-SchwartzMacPherson et homologie d'intersection. C. R. Acad. Sci. Paris Sér. I Math., 314(8):625-628, 1992.

[KZ01] Maxim Kontsevich and Don Zagier. Periods. In Mathematics unlimited_2001 and beyond, pages 771-808. Springer, Berlin, 2001.

[Lev08] Marc Levine. Six lectures on motives. In Some recent developments in algebraic K-theory, volume 23 of ICTP Lect. Notes, pages 131-227. Abdus Salam Int. Cent. Theoret. Phys., Trieste, 2008.

[LL03] Michael Larsen and Valery A. Lunts. Motivic measures and stable birational geometry. Mosc. Math. J., 3(1):85-95, 259, 2003.

[MA] Pedro Morales-Almazán. Grothendieck ring class of Banana and Flower graphs. arXiv:1208.4129.

[Mac74] Robert D. MacPherson. Chern classes for singular algebraic varieties. Ann. of Math. (2), 100:423-432, 1974.

[Mar10] Matilde Marcolli. Feynman motives. World Scientific Publishing Co. Pte. Ltd., Hackensack, NJ, 2010.

[Pat10] Eric Patterson. On the singular structure of graph hypersurfaces. Commun. Number Theory Phys., 4(4):659-708, 2010. 
[PP01] Adam Parusiński and Piotr Pragacz. Characteristic classes of hypersurfaces and characteristic cycles. J. Algebraic Geom., 10(1):63-79, 2001.

[Sch65a] M.-H. Schwartz. Classes caractéristiques définies par une stratification d'une variété analytique complexe. I. C. R. Acad. Sci. Paris, 260:3262-3264, 1965.

[Sch65b] M.-H. Schwartz. Classes caractéristiques définies par une stratification d'une variété analytique complexe. II. C. R. Acad. Sci. Paris, 260:3535-3537, 1965.

[Sch11] Oliver Schnetz. Quantum field theory over $\mathbb{F}_{q}$. Electron. J. Combin., 18(1):Paper 102, $23,2011$.

[SMJŌ77] Mikio Sato, Tetsuji Miwa, Michio Jimbo, and Toshio Ōshima. Holonomy structure of Landau singularities and Feynman integrals. In Proceedings of the Oji Seminar on Algebraic Analysis and the RIMS Symposium on Algebraic Analysis (Kyoto Univ., Kyoto, 1976), volume 12, pages 387-439, 1976/77.

[Sta98] Richard P. Stanley. Spanning trees and a conjecture of Kontsevich. Ann. Comb., 2(4):351-363, 1998.

[Ste98] John R. Stembridge. Counting points on varieties over finite fields related to a conjecture of Kontsevich. Ann. Comb., 2(4):365-385, 1998.

[Str11] Judson Stryker. Chern-Schwartz-MacPherson classes of graph hypersurfaces and Schubert varieties, 2011.

[Vak06] Ravi Vakil. Murphy's law in algebraic geometry: badly-behaved deformation spaces. Invent. Math., 164(3):569-590, 2006.

[Web12] Andrzej Weber. Equivariant Chern classes and localization theorem. J. Singul., 5:153-176, 2012.

[Zee10] A. Zee. Quantum field theory in a nutshell. Princeton University Press, Princeton, NJ, second edition, 2010.

Mathematics Department, Florida State University, Tallahassee FL 32306, U.S.A.

E-mail address: aluffi@math.fsu.edu 